\title{
ANTONIO GREGORIO ROSELL Y VICIANO (CA. 1748-1829): INSTITUCIONES MATEMÁTICAS (1785) \\ Instituciones Matemáticas (1785), by Antonio Gregorio Rosell y Viciano (ca. 1748-1829)
}

\author{
Luis ESPAÑOL GONZÁLEZ \\ Universidad de La Rioja \\ luis.espanol@unirioja.es \\ Juncal MANTEROLA ZABALA \\ Universidad del País Vasco/Euskal Herriko Unibertsitatea \\ mariajuncal.manterola@ehu.es
}

Fecha de recepción: 03/10/2020

Fecha de aceptación definitiva: 31/03/2021

RESUMEN: La Imprenta Real publicó en 1785 Instituciones Matemáticas de Antonio Rosell, catedrático desde enero de 1772 en los Reales Estudios de San Isidro en Madrid. El autor anunció dos volúmenes, el primero contiene la aritmética numérica y los principios del álgebra, pero el segundo quedó inédito. Rosell afirma que quiere escribir un «texto de matemática pura» dirigido a «matemáticos de profesión». Presentamos una descripción detallada del libro, precedida por indicaciones biográficas sobre el autor y aspectos generales de la obra citada.

Palabras clave: historia de las matemáticas; siglo XVIII; España; enseñanza superior; libros de texto.

ABSTRACT: The Royal Printing Office published in 1785 Instituciones Matemáticas by Antonio Rosell, professor since January 1772 at the Royal Studies of San Isidro in Madrid. The author announced two volumes, the first containing numerical arithmetic and the principles of algebra, but the second was unpublished. Rosell 
claims that he wants to write a "textbook on pure mathematics" for "mathematicians by profession». We present a detailed description of the book, preceded by some biographical notices about the author, and general aspects of the cited work.

Key words: history of mathematics; 18th century; Spain; higher education; textbooks.

\section{INTRODUCCIÓN}

Aunque no disponemos de una biografía cabal de Antonio Rosell y Viciano, encontramos datos sobre él en publicaciones varias ${ }^{1}$, a los que añadiremos algunos más ${ }^{2}$.

En este trabajo nos ocuparemos tan solo de la actividad de Rosell como matemático, ejercida durante unos años de juventud y madurez, en la que destaca su obra principal Instituciones Matemáticas (1785), el libro de texto asociado al desempeño, entre 1772 y 1794, de una cátedra en los Reales Estudios de San Isidro, centro público de enseñanza en la capital. En este centro, Rosell se afirmó como un "matemático de profesión" que quiso escribir un "texto de matemática pura» para formar matemáticos como él. Dejó la cátedra en Madrid por razones de salud y volvió a su ciudad natal, Valencia, como comisario de guerra -función que había simultaneado en modo honorario con la cátedra-. Allí vivió más de dos décadas ejerciendo su cargo hasta la jubilación y ocupado en actividades diversas como erudito local. Este periodo final de su vida, aunque dilatado (1794-1829), apenas será considerado en lo que sigue.

En primer lugar, ofreceremos ciertos rasgos biográficos de Rosell relacionados con su formación y actividad como matemático durante el último tercio del siglo XVIII. Luego, nos centraremos en la descripción de su obra antes citada y que aparece en el título, atendiendo primero a cómo la ideó y planificó, para describir después su realización efectiva, que conocemos parcialmente porque no pudo concluirla como la tenía proyectada. Solo pasó por la imprenta el primer tomo, que contiene la aritmética numérica y los principios del álgebra, pero quedó

1. Sempere y Guarinos, Juan. Ensayo de una biblioteca española de los mejores escritores del reinado de Carlos III. Madrid: Imprenta Real, 1789, tomo V, pp. 51-55; FusTER Y TARONCHER, Justo Pastor. Biblioteca Valenciana de los Escritores que florecieron hasta nuestros dias y de los que aun viven. Con adiciones y enmiendas a la de D. Vicente Ximeno. Valencia: Imprenta y Librería de Ildefonso Mompié, 1830, pp. 470-471; Simón Díaz, José. Historia del Colegio Imperial de Madrid. Madrid: Instituto de Estudios Madrileños, CSIC, 1959, tomo II, p. 77; AlBIÑANA HuERTA, Salvador. La Universidad de Valencia y la ilustración en el reinado de Carlos III. Tesis doctoral. Valencia: Universidad de Valencia, 1987, p. 342; Díe Fagoaga, Gonzalo. Francisco Verdejo, un mathematico olvidado. Bubok Publishing S.L., 2010, pp. 59-60, 97, 105, 206-215. Esta última referencia aporta numerosos datos sobre personas y actividad en los Reales Estudios de San Isidro mientras fueron catedráticos sucesivamente Antonio Rosell y Francisco Verdejo.

2. Citaremos documentos de: Archivo General Militar de Segovia (AGMS), Archivo General de Simancas (AGS), Archivo Histórico Nacional (AHN) y Archivo de la Universidad de Valencia (AUV). 
inédito el segundo, que debía estar dedicado a lo más notable de su tiempo, la geometría analítica y el cálculo infinitesimal con aplicaciones a la mecánica.

\section{Detalles Biográficos de ANTONio Rosell}

Antonio Rosell nació en Valencia hacia 1748, años después de que sus padres, José y María Clara, hubieran tenido al menos otros dos hijos viviendo en Castellón de la Plana: Basilio Tomás y Manuel. Tuvo un tercer hermano, Martín Tadeo, del que no conocemos las fechas de vida. Basilio fue un fraile agustino que llegó a prior del convento de Nuestra Señora de Aguas Vivas en Carcagente (Valencia), Manuel fue canónigo de la Real Capilla de San Isidro de Madrid, Martín Tadeo fue profesor de matemáticas en el Real Seminario de Nobles de Madrid ${ }^{3}$. Su nacimiento tuvo lugar reinando el segundo Borbón, Fernando VI, hacia la mitad del periodo 1743-1752 durante el cual el marqués de la Ensenada fue el poderoso secretario de Hacienda, Guerra, Marina e Indias, llevando a cabo una eficiente política científica ${ }^{4}$ que propició un impulso de la matemática española reforzado durante el reinado de Carlos III, iniciado cinco años después de la caída de Ensenada. Estas transformaciones se producían mientras Rosell iba creciendo en su ciudad natal, en cuya universidad ingresó el curso 1765/66, obteniendo el grado de Bachiller en Filosofía en 1768.

A falta de documentación específica sobre su nacimiento, este se fecha a partir de la información que suministra el documento relativo a su ingreso en la Universidad de Valencia, donde se indica que estaba "aprobado en Latinidad" y tenía 17 años cuando comenzó estudios de "Filosofía tomística» en Valencia el día de "San Lucas de 1765» (18 de octubre) para terminar en "San Juan de 1766» (24 de junio) bajo la dirección del «Dr. D. Josef Ivañez Falomir»"

Otro documento no hecho público hasta ahora ${ }^{6}$ explica su formación en la Universidad de Valencia antes de concurrir a la cátedra en los Reales Estudios de San Isidro. En su ciudad natal, con fecha 31 de agosto de 1770, dirigió una súplica al rector de la universidad, Francisco Antonio Cebrián y Valdá, solicitando la certificación de los datos curriculares que exponía en su escrito, a lo que el rector accedió. El escrito iba acompañado de las declaraciones juradas ante Joseph Miñana de dos testigos, ambos valencianos, Francisco Escoin y Joseph Regalado,

3. Un apunte bibliográfico de A. Rosell aparece en AgUiLAR PiÑAl, Francisco. Bibliografía de autores españoles del siglo XVIII. Tomos I-X. Madrid: CSIC, Instituto Miguel de Cervantes, 1981-2001, tomo VII, p. 312. Diseminados por el resto de los tomos hay registros de otros autores en los que aparece citado Rosell. Sus tres hermanos son mencionados también en esta obra.

4. Véase Gómez URdáÑEz, José Luis. El proyecto reformista de Ensenada. Lleida: Ed. Milenio, 1996.

5. AUV Arxiu General, llibre 006, ff. 47r.-47v. En AUV Arxiu General, llibre 032, ff. 267r.-268r. consta la obtención del grado de Bachiller en Filosofía.

6. AUV Arxiu General Caixa n. ${ }^{\circ}$ 0883-33. 
que afirmaban conocer al solicitante y haberle acompañado en las actividades curriculares que esgrimía. Rosell pedía que se certificara que
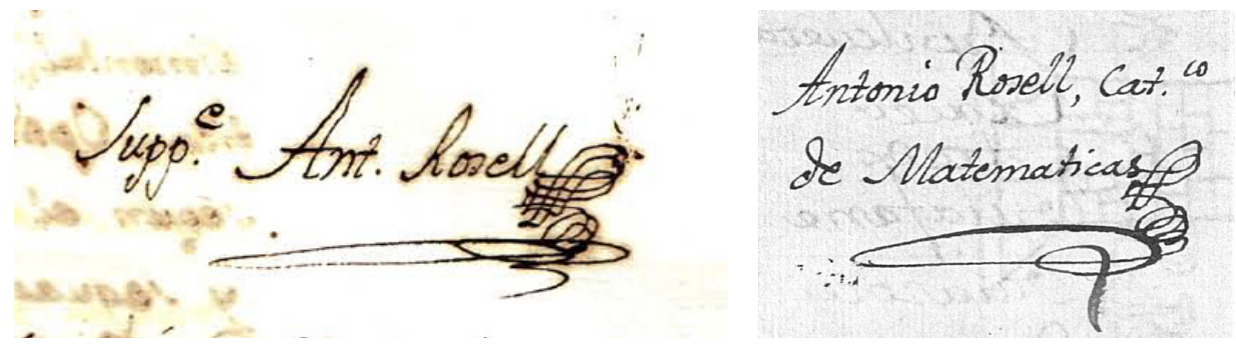

Fig. 1 Firmas Rosell; izda. 1770, dcha. 1777.

Fuentes: izda. AUV Arxiu General Caixa n. ${ }^{\circ}$ 0883-33, dcha. AHN. Leg. 5540, Exp. 46.

[...] empezó el estudio de la Filosofia Tomistica en el año 1765 bajo la direccion del D. ${ }^{\mathrm{r}}$ D. ${ }^{\mathrm{n}}$ Josef Ivañez: que cumpliò en los tres años de dicho estudio, acudiendo a mas de la Aula a dos Academias publicas, y otras privadas de otra Facultad : que finalizado el curso obtuvo los Grados de Bachiller y Maestro en Artes, defendiendo para este, a mas del curso regular que se dicta; todas las proposiciones de Arithmetica, y Geometria pertenecientes a la Fisica Experimental y la Mecanica, Estatica, Hydrostatica, Optica, Dioptrica, Catoptrica, y Astronomia segun el systema del Cavallero Newton, y sequaces : que tiene dos años de Estudio de Teologia : y finalmente, que haviendo firmado en el año 1768 a la Oposicion de la Catedra de Matematicas, vacante por ascenso del Ilmo S. ${ }^{\text {or }} \mathrm{D} .{ }^{\mathrm{n}} \mathrm{Fr}$. Rafael Lassala Obispo de Adramita, se desempeñó cumpliendo en todo lo que debia egercitarse para dicha Oposición : arguiendo tres argumentos de a media hora, respondiendo a seis, tres por la mañana y tres de a media hora por la tarde que le arguieron sus coopositores, y leiendo a las 24 una hora de dos puntos uno de Geometria y otro de Astronomia; haviendo formado para dichos actos un Cuerpo de conclusiones en las que, a mas de tratarse por extenso casi todo lo que debía enseñar el Catedratico segun constituciones, y arreglarlo al gusto moderno, defendia en una conclusion varíos tratados de Matematicas de Wolfn como son: Arithmetica, Geometria, Trigonometria, Mecanica, Hydrostatica, Aerometria, Hydraulica, Optica, Catoptrica, Dioptrica, Perspectiva, Astronomia, Geografia, Chronologia, y Gnomonica.

En la oposición a la cátedra de la Universidad de Valencia no tuvo éxito, pero en aquellos años de cambio y apertura de nuevos horizontes matemáticos en España, a Rosell, que había alcanzado el grado de Maestro en Artes en la Universidad de Valencia el año 1769, se le abrían nuevas oportunidades cuando en España aparecía un incipiente profesorado de matemáticas profesional7 ${ }^{7}$, al margen de los

7. Véase ViÑao Frago, Antonio. «Disciplinas académicas y profesionalización docente: los Reales Estudios de San Isidro (1770-1808)». En FeLL, Ève-Marie y GuEREÑA, Jean-Louis (dirs.). L'Université en 
tradicionales eclesiásticos y militares. La expulsión de los jesuitas en 1767 supuso el cierre de los Reales Estudios del Colegio Imperial, que fue reabierto en 1770 e inaugurado un año después, con el nombre de Reales Estudios de San Isidro y convertido en un centro gratuito del Estado que necesitó reclutar profesores. Se convocaron dos oposiciones públicas a un tiempo para cátedras de matemáticas, figurando en el tribunal Benito Bails y Francisco Subirás y Barra, catedráticos desde 1768 en la Real Academia de Bellas Artes de San Fernando. Se celebraron a lo largo de 1771, la primera fue ganada por Joachin de León y la segunda por Antonio Rosell ${ }^{8}$. Este último expuso en la oposición una disertación titulada De la dioptrica analítica, la leyó en latín como era preceptivo, pero no usual.

El 18 de noviembre de 1773, los catedráticos noveles León y Rosell hicieron llegar al Consejo de Castilla una propuesta muy detallada para ampliar de dos a cuatro, uno por año, los cursos de matemáticas en los Reales Estudios, junto con la dotación de dos nuevas cátedras y otro profesorado. Estas propuestas no prosperaron, como tampoco otras dos que acompañaban con mayor alcance institucional. Solicitaban, con detalles sobre su constitución, la creación de una "Junta de Matematicos baxo la imediata proteccion de S. M., é independiente de todas las Universidades, y Estudios del Reyno", con el fin de "fomentar y perfeccionar el estudio de las Matematicas en España", regulando en particular los "grados de Bachiller y Doctor en Matematicas». Pedían también "dar principio á una Real Academia de Ciencias Española», señalando que aun si esta llegara a existir, "es siempre necesaria la Junta de Matematicos por ser muy distinto su obgeto aunque contribuya al mismo fin»?

En 1775, redactó Demonstracion de las causas que concurrieron á los daños y ruinas de las obras del Prado nuevo de Madrid en la tarde del 23 de Setiembre de 1775, y modo de precaverlas en adelante, un informe técnico que incluye «un Plano del Paseo con indicación de los sitios dañados por las lluvias y aconseja métodos preventivos basados en la experiencia, el cálculo y la dinámica» ${ }^{10}$. El informe tuvo cierta repercusión y se incluyó como tema en ejercicios públicos de estudiantes. De uno de ellos da noticia La Gaceta de Madrid (17 de noviembre de 1780, n. ${ }^{\circ}$ 92). La Real Sociedad Bascongada celebró en Vitoria su asamblea general, del 27 de septiembre al 2 de octubre de 1780, llevándose a cabo «3 sesiones públicas para instruir al concurso de sus operaciones y de los progresos de sus Seminaristas». En estas tres sesiones públicas se examinó, entre otros, Joseph Maria de

Espagne et en Amérique Latine du Moyen Âge à nos jours. II: Enjeux, contenus, images. Tours: Presses universitaires François-Rabelais, 1998. Edición disponible en línea (generada el 16 de abril de 2021): http://books.openedition.org/pufr/5982.

8. AGS, GYJ, LEG. 972: Registro de opositores a las cátedras, su procedencia, etc. Rosell fue el segundo en la terna que el tribunal propuso al Consejo, pero fue quien recibió el nombramiento del rey.

9. Véase Simón Díaz, José. "Los Reales Estudios de San Isidro: Nuevas noticias». Anales del Instituto de Estudios Madrileños. Madrid: Instituto de Estudios Madrileños, CSIC, 1973, IX, pp. 323-340.

10. Simón Díaz (1959), op. cit., 77. 
Lanz $^{11}$, alumno de la clase de Matemáticas del Seminario Patriótico, quien hubo de contestar entre las cuestiones planteadas preguntas acerca de esta obra de Rosell:

[...] sostuvo $1 .^{\circ}$ todos los tratados que se contienen en los principios de Matemática de D. Benito Bails hasta la Mecánica inclusive, con varias proposiciones y fórmulas de sus elementos: $2{ }^{\circ}$ la Estática y Dinámica que se encierran en el Exâmen marítimo de D. Jorge Juan: $3 .^{\circ}$ la Artillería analítica: $4 .^{\circ}$ la sublime teoría de los muros, arcos y bóvedas de Juan Muller: $5 .^{\circ}$ la demostración hecha por D. Antonio Rosell Viciano de las causas que concurrieron á los daños y ruinas de las obras del Prado nuevo de Madrid en la tarde del 23 de Setiembre de 1775.

El principal legado de la actuación de Rosell en los Reales Estudios es el primer tomo de Instituciones Matemáticas, pero también dio a la luz un año antes un librito escolar titulado La Geometría de los niños ${ }^{12}$. Se trata de una obra de 104 páginas en la que se plantea una lista de preguntas y respuestas que el estudiante debe aprender de memoria y comprender visualmente con la ayuda de cuatro láminas desplegables con figuras. Pretende el autor «aficionar á los niños al estudio de las Matemáticas, y disponerles para el de otras facultades, y exercicio de las Artes".

Otros datos de su desempeño académico son los «egercicios» públicos de sus alumnos más destacados, la "censura" de manuscritos para imprimir y la participación en tribunales de oposiciones. Presidió numerosos ejercicios públicos durante la primera década en la cátedra ${ }^{13}$, con algunos estudiantes que alcanzaron fama, como Josef Maria de Mendoza y Ríos (1774) y Agustín de Betancourt y Molina (1780).

Su primera censura documentada ${ }^{14}$ es de 1777, cuando Rosell avaló imprimir la obra Elementos de matemática pura (1778, dos tomos, uno de aritmética y otro de geometría) de Carlos Lemaur, "Coronel e Ingeniero en gefe de los Reales Exercitos». Pensaba el autor que su obra estaba realizada "con un methodo nuevo cuya impresion juzga util a la Enseñanza publica». La solicitud de impresión llegó a Manuel de Villafañe, director de los Reales Estudios, quien pasó el encargo a Rosell. Un tiempo después, Lemaur pidió que se le recordara al censor la tarea que tenía pendiente para que él pudiera asistir a la impresión de su obra durante

11. Véase Caballer Vives, María Cinta. El Álgebra en la Enseñanza Secundaria en España (1836-1936). Tesis doctoral dirigida por Josep Llombart Palet. Leioa: UPV/EHU, 2006. En p. 203, nos da noticia de que Joseph Maria de Lanz y Zaldivar fue el «autor de los planes de estudios y persona encargada de seleccionar al profesorado cuando Agustín de Betancourt fundó la Escuela de Ingenieros de Caminos".

12. Rosell Viciano, Antonio Gregorio. La Geometría de los Niños. Madrid: Imprenta Real, 1784. El autor aparecía en la portada solo con iniciales: «D. A. R. C. D. M.» Léase: Don Antonio Rosell Catedrático de Matemáticas.

13. Tenemos noticia de 18 entre 1773 y 1786 en Agullar Piñal, op. cit.; Díe FagoAgA, op. cit., y MERCURIO DE ESPAÑA, agosto de 1786. Madrid: Imprenta Real, pp. 359-360.

14. AHN, leg. 5540, exp. 46. 
su estancia en la Corte ${ }^{15}$. Rosell se apresuró entonces a emitir una breve censura el 8 de marzo de 1777, sin incluir un análisis crítico de la obra se limitó a declarar:

[...] nada hallo en ellos que deba retardar la licencia de impresion que se solicita ; antes bien juzgo que son de los mas dignos de la luz publica que pueden ofrecerse en esta materia, por la novedad, sublimidad, y maestria con que están díspuestos.

Cuando Joachin de León falleció en 1778, Rosell le sustituyó mientras se convocaba la oposición, en la que actuó como examinador ${ }^{16}$. Con el nuevo catedrático, Vicente Durán y Sacristán, realizó en los años ochenta tres censuras conjuntas a manuscritos relacionados con la circunferencia y el círculo, las tres fueron desfavorables. En 1787, Rosell participó también ${ }^{17}$ en la oposición que se celebró en los Reales Estudios de San Isidro para cubrir una cátedra de matemáticas en el Colegio de San Telmo de Sevilla. Esta oposición es la última noticia que tenemos de una actividad académica de nuestro personaje, si bien hay constancia de que el 15 de diciembre de 1792 insistió en una propuesta que ya había elevado junto con su colega León en 1773; esta vez, Rosell presentó Apuntamientos para establecimiento de Academia R.l de Ciencias en la Corte de Madrid, academia que tendría una sección de matemáticas y filosofía ${ }^{18}$.

Una enfermedad que venía arrastrando desde 1783 obligó a Rosell a estar de baja cada vez más a menudo, siendo sustituido entonces por Joseph Miguel de Sarasa ${ }^{19}$. Al no tener la enfermedad un final previsible, las autoridades de los Reales Estudios prepararon su relevo: en 1793 recibió Rosell el nombramiento real de comisario de guerra con destino en Valencia, al año siguiente solicitó la baja como catedrático y partió hacia su ciudad natal. De su actividad en Valencia durante los últimos años del siglo XVIII y primeros del XIX, solo dejaremos constancia de un Tratado de la Tactica de los Regimientos de Infanteria. No se conoce el manuscrito, pero está documentado que solicitó permiso real para imprimirlo. El inspector general de Infantería Francisco Javier de Negrete recibió el 12 de marzo de 1801, a través del «Ex. ${ }^{\text {mo }}$ S. ${ }^{\text {or }} \mathrm{D} .{ }^{\mathrm{n}}$ Josef Antonio Caballero ${ }^{20}$ el encargo real» de censurar el manuscrito. El censor presentó a Rosell como "Comisario de Guerra honorario",

15. Lemaur viajaba con frecuencia por el territorio nacional para dirigir importantes obras de ingeniería. Véase CAPEL SAEZ, Horacio; SÁNCHEz JimÉnEZ, Joan Eugeni y MoncADA MayA, Omar. De Palas a Minerva. La formación científica y la estructura institucional de los ingenieros militares en el siglo XVIII. Barcelona: Serval/CSIC, 1988.

16. Díe Fagoaga, op. cit., pp. 83-84.

17. Véase Memorial Literario, Instructivo y Curioso de la Corte de Madrid, 1787, tomo XI, número LXI. Madrid: Imprenta Real, pp. 451-452.

18. Véase JACOBS, Helmut C. «Utopías de la organización e institucionalización de las artes y ciencias en la época de la ilustración: Los proyectos de fundación de academias». Anejos de Dieciocho, 2019, 5, Spring. Universität Duisburg-Essen, pp. 141-161.

19. Era capitán y profesor del Real Observatorio de Madrid cuando publicó Sinopsis de Astronomía teórica (1802) y unas Tablas astronómicas (1816).

20. Secretario de Estado de Gracia y Justicia de Carlos IV. 
realizó un amplio y detallado examen de la obra a lo largo de 20 páginas y como colofón concluyó el 26 de marzo de $1802^{21}$ :

Esto es lo que me ha parecido mas notable de quanto contienen las obras indicadas, y he omitido hacer mas larga mención de ellas por parecerme que basta lo expuesto para dar un pleno conocim..$^{\text {to }}$ de su irregularidad, desconcierto y confusion. Cada pagina publica y pone de manifiesto la enfermedad de que adolece el autor, y no pueden leerse sin la tristeza que engendra el considerar ser produccion de un sugeto que tubo buen talento. Considero que resultaria un notable perjuicio á su credito é intereses si se accediese á su suplica: lo primero por hacer patentes y publicos los desconciertos y delirios de una cabeza enferma; y lo segundo por que no podria reintegrarse de los gastos de la impresión.

La ciencia militar interesa directamente al Gobierno, y pudiera dar una mala idea de su ilustracion si con su permiso se publicasen esas obras. El mismo interesado se avergonzaria de que hubiesen visto la luz publica si tubiese la fortuna de llegar á recobrar su buen juicio. Me parece pues digno de que se le guarden los respetos que se le deben; en cuya atención, y a lo que queda dicho anteriormente soy de dictamen de que baxo algun pretexto honesto se le niegue lo que solicita.

Con su mala salud a cuestas, descrita por Negrete de un modo rotundo y a la vez amable, Rosell permaneció activo en la vida cultural valenciana, colaborando como erudito local en el Diario de Valencia, periódico de los años 1790-183422, y publicando un par de libros. Su fallecimiento fue comunicado oficialmente:

Ordenacion en Gefe del distrito militar de la Capitania Gen. ${ }^{\text {al }}$ de Valencia= El Comisario de Guerra jubilado D. Antonio Rosell fallecio en esta Capital en la mañana de ayer; lo cual aviso a V.S. para su devido conocimiento=Dios guarde a V.S. m. a. Valencia 24 de Marzo de 1829= Juan Gomez Landero $=$ Sr Intend.$^{\text {te }}$ Gen..${ }^{\text {al }}$ del Ejto. ${ }^{23}$.

\subsection{El libro de texto de Rosell}

La enseñanza de las matemáticas en los Reales Estudios de San Isidro, que se prolongaba durante dos años académicos con un programa que comprendía aritmética, álgebra, geometría, trigonometría, curvas, cálculo diferencial e integral y mecánica, fue iniciada por dos catedráticos que se alternaban cada año en la docencia para mantener los mismos alumnos durante los dos cursos consecutivos. Rosell comenzó su tarea docente en 1772 explicando el primer curso según Elementa matheseos universae (4 vols. 1713-1715) de Christian Wolff. Así se acredita en el Egercicio de Matematicas de Pedro Vicente Giner $^{24}$, de 4 de julio de 1773,

21. AGMS 1. $/ \mathrm{R}-3099$.

22. Accesible en RODERIC, el repositorio de contenido libre de la Universidad de Valencia.

23. AGMS 1. $/$ R-3099.

24. GINER, Pedro Vicente. Egercicio de Matematicas. Madrid: Joachin Ibarra Impresor de Cámara de S. M., 1773, p. 38. 
el primero de los que presidió nuestro joven catedrático. Giner termina con una "Advertencia» en la que se lee ${ }^{25}$ :

En los nueve meses que se han empleado en el estudio de la Aritmetica, Geometría, y Trigonometría, a que está ceñido este Egercicio, se han explicado estos Tratados con la misma extension que se hallan en los Elementos de Matemáticas del celebre Cristiano Wolf, a quien se sigue en estos Estudios; [...]

Por otra parte, en el Egercicio de Matemáticas de segundo curso realizado por Josef María de Mendoza el 31 de diciembre de 1774, también presidido por Rosell, hay un apartado sobre mecánica, en cuyo preámbulo se indica que «ninguna obra pareció mas proporcionada al intento, que la Mecánica que se halla en el Exâmen Marítimo [...] es pues la que vamos á proponer ${ }^{26}$, de modo que Rosell explicaba la principal aplicación del cálculo infinitesimal siguiendo la reconocida obra de Jorge Juan y Santacilia.

El inicio de las clases de Rosell siguiendo a Wolff coincidió con la aparición de Elementos de Matemáticas en 1772 (11 volúmenes completados en 1783), la obra monumental de Benito Bails que fue la referencia para la matemática española del último cuarto del siglo XVIII. Consciente de la necesidad de un manual más reducido para la enseñanza, publicó Principios de Matemáticas (3 tomos, 1776), anunciándolos como "extracto" o "compendio" de Elementos. La obra de Bails se convirtió en el texto oficial de matemáticas en los Reales Estudios.

Rosell quiso ser autor de su propio texto, como otros profesores que escribían obras adaptadas a sus cursos, aproximando el contenido a las lecciones realmente impartidas y a las posibilidades de compra de libros por parte de los estudiantes. Como empezó muy novato, necesitó tiempo para componer con criterios propios un manual ajustado a sus cursos; al fin, catorce años después de conseguir la cátedra, en un buen momento para el centro $^{27}$, surgió Instituciones Matemáticas, cuyo primer tomo (aritmética y álgebra) apareció en $1785^{28}$, mientras que el segundo quedó inédito. Instituciones fue el libro de texto para aritmética y álgebra en el primer curso de los Reales Estudios de San Isidro, hasta que Rosell cesó en la cátedra por razones de salud en 1794, a los 46 años.

25. En las citas de Instituciones que daremos entre comillas conservaremos el texto literal del autor, igual que hemos venido haciendo en las citas de otros documentos.

26. De MENDOZA y Ríos, Josef María. Egercicio de Matemáticas. Madrid: Joachin Ibarra Impresor de Cámara de S. M., 1774, p. 20.

27. Según De la FuENTE, Vicente. Historia de las universidades, colegios y demás establecimientos de enseñanza en España. Madrid: Imprenta de la viuda é hija de Fuentenebro, 1889, tomo IV, p. 163, en 1785 los Reales Estudios tenían cerca de 400 matriculados, casi tantos como la Universidad de Alcalá. De ellos, cursaban matemáticas 63, número considerable «que tan escaso había sido en otro tiempo, y eso que decían que España no era tierra de filósofos ni de matemáticos».

28. Rosell Viciano, Antonio Gregorio. Instituciones Matemáticas. Madrid: Imprenta Real, 1785. 
Los libros de texto debían ser propuestos por el director de los Reales Estudios, todavía Manuel de Villafañe, así que fue un reconocimiento para Rosell que su obra sustituyera en parte a la de Bails. Sempere ${ }^{29}$ publicó una reseña que había sido enviada desde Madrid, sin firma, al número de 13 de mayo de 1786 de la revista Efemeridi letterarie di Roma ${ }^{30}$, informando de la publicación de Instituciones y alabando la originalidad del método seguido por el autor, de quien se dice que posee "a fondo le scienze che prende à trattare» $y$ es «allo stesso tempo gran metafisico".

Instituciones se adoptó como texto en el Real Colegio Seminario de San Telmo de Sevilla, según consta en las Ordenanzas fundacionales del centro de 1786, en las que se lee ${ }^{31}$ :

Mientras se publican Instituciones propias de estos estudios, se valdrán los Catedráticos de las obras que sobre dichas materias han dado á luz D. Vicente Tofiño, D. Benito Bails, y D. Antonio Rosell, señalando de comun acuerdo con el Director y los otros Catedráticos las partes que podrán tomarse de cada Autor, lo que convenga suplir, el órden y la distribucion para los dos años.

Esta ordenanza indica que Instituciones de Rosell era el texto para aritmética y álgebra; que la geometría se enseñaría con el Compendio de la Geometría elemental y Trigonometría rectilínea (1771) que Vicente Tofiño de San Miguel Vanderwale preparó en Cádiz para los guardiamarinas, de cuya Academia fue director en 1768-178932; para el resto de las materias matemáticas había que seguir a Bails.

La vigencia de Instituciones de Rosell en los Reales Estudios de San Isidro terminó cuando su autor abandonó la cátedra. Para retirar la obra, el director adujo que contenía errores, aunque no indicó cuáles eran. El catedrático que ocupó su lugar, Francisco Verdejo González (1757-1817), imprimió un libro, pero el director, entonces Estanislao Lugo y Molina, no lo autorizó como texto. Verdejo reclamó sin éxito al Consejo de Castilla, quedando la obra de Bails como referencia oficial única ${ }^{33}$.

29. SEMPERE y GUARINOS (1789), op. cit., p. 53.

30. Sobre las reseñas de textos españoles del dieciocho en la citada publicación véase GimENO PuYol, María Dolores. «Il poco numero dé libri Spagnoli»: La defensa de España en las Efemeridi letterarie di Roma en el último tercio del siglo XVIII". Cuadernos de Historia Moderna, 2019, 44, 1, pp. 181-204.

31. Ordenanzas [1786, artículo CCIV, p. 96].

32. Véase en Manterola Zabala, María Juncal. Las matemáticas en los estudios de náutica en España en el siglo XVIII: estudio comparativo de los libros de texto empleados en la formación de pilotos y guardiamarinas. Tesis doctoral dirigida por Luis Español e Itsaso Ibáñez. Logroño: Universidad de La Rioja, 2016, pp. 203-206 (apuntes biográficos), pp. 324-331 y 412-419 (descripción y análisis de la citada obra).

33. Para más detalles sobre este asunto, véase Díe FAGOAGA (2010), op. cit., pp. 206-215. 
3. DESCRIPCIÓN DE INSTITUCIONES MATEMÁTICAS

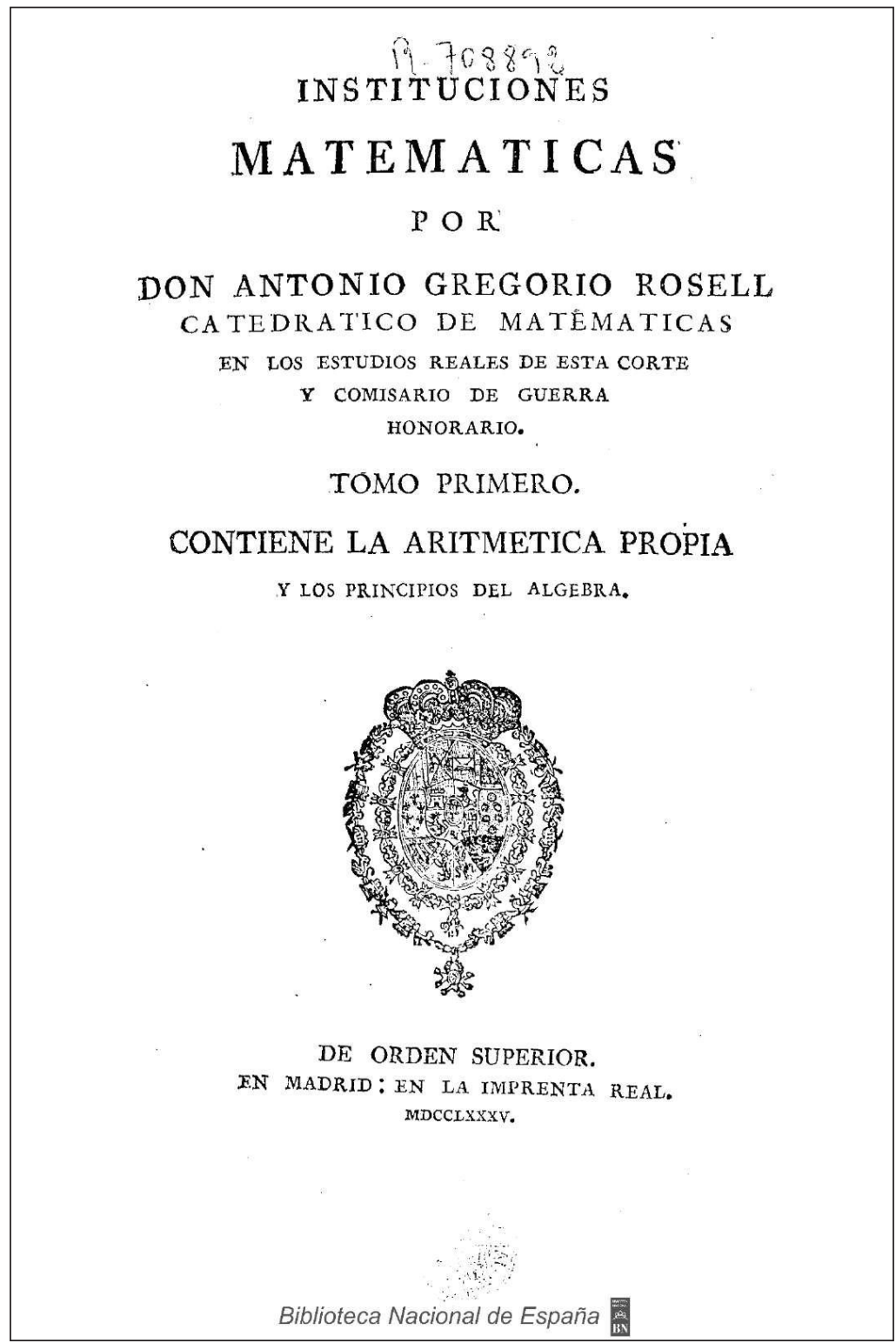

Fig. 2. Portada de Instituciones Matemáticas.

Fuente: BNE 
Se trata de un libro que «contiene la Aritmética propia y los principios del Algebra». El autor dedica la obra al conde de Floridablanca, refiriéndose al texto "que empiezo á publican, pero este tomo fue el único publicado de los dos previstos. Se inicia con 6 páginas sin numerar que contienen la portada y la dedicatoria, a ellas siguen 42 páginas numeradas en romano con textos preliminares, dos más de erratas y otras dos con el índice, estas cuatro sin numerar. A continuación, llega la parte doctrinal de 392 páginas numeradas en arábigo, de las que las 4 primeras contienen una "Introducción». Siguen, para terminar, 16 páginas no numeradas de "Tablas de los números simples y compuestos comprehendidos en 2000, y de los factores ó divisores simples en que se resuelven los compuestos: explicadas $§ 160$, pag. 83".

\subsection{Páginas preliminares}

Instituciones se inicia con dos textos preliminares de notable extensión: un «Prólogo» (pp. I-XXIV) y una "Instruccion proemial» (pp. XXV-XLII). En el primero Rosell expone la visión de las matemáticas y de su enseñanza que guían el diseño de su obra, mientras que en el segundo se ocupa de los métodos usados en las matemáticas, así como de las aptitudes que deben tenerse y de las actitudes que deben adoptarse para estudiarlas.

\subsubsection{Prólogo 34}

Resumimos lo expuesto por Rosell a lo largo de las primeras páginas (I-XI) en estos cuatro aspectos que allí desarrolla: (1) Necesidad de «alterar el plan de instruccion que observaron los antiguos", debido a la aparición del álgebra con su lenguaje simbólico y su aplicación a la geometría. (2) Hay que «formar matemáticos de profesion [...] para que no falten dignos profesores». (3) La aritmética, el álgebra y la geometría se deben enseñar de modo relacionado y con un lenguaje único común para «los que hayan de profesar las Matemáticas». (4) Reducir el contenido a lo fundamental todo lo posible para sustentar el estudio más avanzado y hacerlo acorde con la duración de los cursos.

Después de proclamar la necesidad de disponer de buenos libros elementales, afirma que en matemáticas, "desde que Vieta y Descartes, habiendo promovido la Algebra , comenzaron á aplicarla á la Geometría» se alteró el lenguaje matemático:

Euclides le conoció compuesto solamente del vulgar, de términos facultativos, de números, lineas y figuras, y ahora le vemos enriquecido, y perfeccionado con las letras y signos algebraycos, que han dado muchisima brevedad y claridad á los

34. Rosell Viciano (1785), op. cit., pp. I-XXIV. 
razonamientos, y han facilitado la mayor parte de los descubrimientos, que han extendido los límites de estas Ciencias. [...] Estas apreciables ventajas que nacen de las letras y signos muestran claramente quánto se ha mejorado el lenguage matemático , y quán poderosas razones hay para alterar el plan de instrucción que observaron los antiguos ; formando el sistéma de la Matemática pura con relacion al estado actual del lenguage, como en orden á su edad lo procuró Euclides en sus Elementos.

Acompañando a estos argumentos relativos al punto (1), Rosell cita a pie de página La Logique, ou les premiers développemens de l'art de penser (1780) de Étienne Bonnot de Condillac ${ }^{35}$, donde el abate francés defiende que el álgebra es el lenguaje de las matemáticas, afirmación que discute Rosell sosteniendo que el lenguaje matemático se compone del lenguaje algebraico y también del lenguaje común. Sobre el punto (2), Rosell afirma que muchos autores han escrito textos destinados a militares, marinos, artesanos, etc., entre los que elogia el Cours de Mathématiques a l'usage des Gardes du Pavillon et de la Marine de Étienne Bézout (4 volúmenes, 1764-67); pero esta exposición de la matemática pura como mero recurso para la aplicada le parece insuficiente para profesionales de la matemática ${ }^{36}$ :

Pero claro es, que los Elementos que son buenos para algunos fines particulares, no se puede decir que lo son para formar matemáticos de profesion. Este es un fin superior que abraza los otros, y que por la mayor dificultad que ofrece ha exercitado mas á muchos sábios, siendo al que principalmente deben atender para que no falten dignos profesores que ilustren y adelanten estas Ciencias, y las hagan redundar en beneficio comun. [...] Mas para profesar las Matemáticas es necesario que la enseñanza se ordene desde el principio á la mas cabal inteligencia de los mejores escritos de Matemática pura , que nos han dexado algunos sábios Geómetras , como Newton, Leibnitz, Maclaurin , Euler , Cramer , Clairaut, d'Alembert , y otros. [...] en los [Elementos] que se ordenen á formar perfectos matemáticos [...] importa conservar la exâctitud, y acostumbrar á ella á los jóvenes desde el estudio de los primeros elementos ; pues aunque sea algo mas trabajoso, es medio indispensable para adelantar en esta materia.

Los argumentos respecto al punto (3) comienzan con una crítica a la manera habitual de explicar la aritmética, el álgebra y la geometría ${ }^{37}$ :

35. Rosell cita esta obra reciente de Condillac por la versión original en francés. En 1784 se publicó la traducción española de Bernardo María de Calzada, véase CoBos Bueno, José Miguel y Vallejo Villalobos, José Ramón. "Bernardo María de Calzada y la traducción de la Lógica de Condillac en España». En GonzÁlez Redondo, Francisco de Asís (coord.). Ciencia y Técnica entre la Paz y la Guerra. 1714, 1814, 1914. Madrid: SEHCYT, 2015, pp. 825-832.

36. ROSELl Viciano (1785), op. cit., pp. VI-VIII.

37. Rosell Viciano (1785), op. cit., pp. VIII-IX. 
[...] á pesar del zelo y diligencia que han puesto algunos doctos Profesores en la formacion de los Elementos que tenemos dirigidos á la enseñanza de los matemáticos, se hallan todavía varios defectos que perjudican la mejor instruccion, é impiden el adelantamiento. Porque la Aritmética propia, y los principios del Algebra, y la Geometría elementar se miran en el modo de tratarlas casi como independientes entre sí. [ ...] y no pueden formar el debido sistema de la Matemática pura. [...] de los débiles fundamentos de la Aritmética se sigue la poca firmeza de los principios del Algebra; y que la Geometría se suele facilitar haciendola perder gran parte de la exâctitud que siempre ha resplandecido en ella.

Como excepción a esta crítica general, Rosell destaca «al Abate Bossut, y al Brigadier Don Carlos Lemaur, por lo que se han distinguido en estos ultimos tiempos en procurar la exâctitud ; el primero en sus Tratados de Aritmética y Algebra , y el segundo en sus Elementos de Algebra y Geometría». En cuanto al punto (4), indica (pp. IX-XI) que se aparta «del modo de pensar de aquellos que les parecen utiles los Cursos extensos de la facultad". Su objetivo es lograr un contenido esencial para que los estudiantes "quanto antes se hallen en estado de entender las demas obras , y de poder formar al paso que las estudien un conocimiento histórico y mas extenso de las Matemáticas [...] indispensable á qualquiera que haya de profesar la Facultad [...]" En cuanto a la extensión , quiere "tratar la Matemática pura , y las partes de la mixta que comprehenden la Mecanica, Optica, y Astronomia, con una extension que puedan los de mediano talento concluir su estudio en quatro años , que es tiempo suficiente para cursar estas Ciencias , y conforme al que se suele emplear en otras Facultades ${ }^{38}$.

El prólogo avanza explicando el plan trazado para la obra , que resumimos en estos criterios: (A) Buscar principios generales para los más útiles conocimientos y ordenarlos del modo más natural con igual lenguaje y exactitud, evitando repeticiones. (B) El principio más fácil y general de las matemáticas es la noción de las relaciones de la cantidad. (C) Se deben usar indistinta y simultáneamente los métodos analítico y sintético.

Respecto al criterio (A), Rosell ya había criticado (nota al pie de la página XI) «el Curso completo que publicó en 1774 el Abate Sauri», por "extractar y enlazar cosas derivadas de muy diferentes principios, que no se pueden unir sino con palabras»; por el contrario, él pretende ${ }^{39}$ :

[...] refundir los Elementos de la Matemática pura, buscar los principios mas generales con relación á los mas utiles descubrimientos, ordenarlos del modo mas natural, derivando de ellos los demas, y escusar repeticiones, usando en todo

38. Se impartían dos cursos de matemáticas, uno por año, así que la mención a cuatro años podría deberse a que los estudiantes de mediano talento tardaban cuatro años en terminarlos o bien a que Rosell seguía manteniendo la pretensión de cuatro cursos anuales de matemáticas solicitada junto con Joachin de León en 1773.

39. Rosell Viciano (1785), op. cit., p. XII. 
el mismo lenguage facultativo, de manera que parezca que toda la Ciencia se ha inventado de una sola vez, y que en todo se halle igual exâctitud.

Euclides lo hizo con la matemática y el lenguaje geométrico de aquel tiempo y Rosell pretende hacerlo con la matemática del nuevo tiempo ilustrado provista del lenguaje algebraico. El matemático valenciano entra de lleno en el criterio (B) sobre «las relaciones que podemos determinar, contando , midiendo , ó pesando" ${ }^{40}$ :

[...] tomo por principio mas facil y general la nocion de la relacion ; porque tratando las Matemáticas de las relaciones de la cantidad, dicha nocion debe reynar en todas ellas. De la nocion de la relacion nace inmediatamente la del número ; y la division de los números en determinados, é indeterminados, dá los dos ramos de Ciencia que constituyen la Aritmética propia, y el Algebra. [...] tengo por indispensable anteponer la Aritmética propia al Algebra, y hacer formar en aquella las primeras nociones de esta. [...] considerando á la Aritmética numérica como parte del Algebra , comprehendo las dos baxo el nombre de Aritmética universal, segun lo hizo el Caballero Newton.

Es bien conocido que la contraposición clásica entre análisis y síntesis distingue el análisis como método para alcanzar el conocimiento, de invención o de resolución de problemas, mientras que la síntesis es la exposición sistemática del conocimiento alcanzado. Estas ideas están presentes en el criterio (C) , referido al pensamiento de Rosell sobre la dualidad análisis-síntesis en las matemáticas (pp. XV-XIX). Arranca así su digresión:

Muchos distinguen dos métodos en las obras matemáticas ; llamando sintéticas á las que carecen de expresiones algebraycas, y van acompañadas de los nombres, difinicion, corolario, teorema , \&c. y analíticas á aquellas en que se trata la Ciencia sin estos nombres, y con el uso del Algebra. Pero se advertirá el poco fundamento é inutilidad de estas reglas, considerando que no hay escrito á cuyas partes no se les puedan dar dichos nombres, y que se pueden tratar muchas cosas analíticamente sin cálculo alguno ; y al contrario, otras sinteticamente por medio de las expresiones algebraycas ; pues los cálculos algebraycos no son sino modos abreviados de expresar los razonamientos, lo qual pertenece al lenguage, y de ninguna manera constituye el método.

Rosell vuelve a citar la Logique de Condillac, en la que el autor francés se muestra partidario del método analítico que favorece la invención. Pero Rosell señala que para Condillac el método de invención no es solo el analítico «de resolucion ó de descomposicion», pues se van «alternando las descomposiciones y composiciones hasta hacer en el entendimiento una composición total de las partes del obgeto , conocidas sus relaciones». Concluye que, aunque el análisis deba prevalecer "porque de las buenas descomposiciones resultan con mucha facilidad las composiciones", prefiere proponer para el método de invención el nombre de «alternado de analítico

40. Rosell Viciano (1785), op. cit., pp. XII-XV. 
y sintético"; este es el método, dice, que el profesor de matemáticas debe seguir y así lo hará él, empleando «analisis ó sintesis segun mas convenga para hacer formar con claridad el sistema de la Ciencia, y para que todo quede demonstrado con la debida generalidad y exactitud». Operar así le ha obligado a ser original , a:

[...] tratar de nuevo casi todo lo que comprehende este tomo, y comprehenderá el siguiente, demonstrando muchas cosas que hasta ahora no he visto demonstradas , y dando á otras el lugar que les pertenecia.

La parte final del prólogo está destinada a explicar su modo de exponer: dar a conocer la necesidad de lo que se estudia , uso equilibrado de la terminología, tendencia a usar párrafos cortos, explicaciones y demostraciones breves, necesidad de distinguir principios, fundamentos y consecuencias, "proponiendo las verdades que se han de deducir antes de inferirlas», al uso de las citas internas en la obra , al importante papel de los ejemplos para ayudar a la adquisición de las ideas abstractas, etc.

\subsubsection{Instrucción proemial ${ }^{41}$}

Este proemio es menos original que el prólogo. Lo escribe para que «los principiantes tengan formadas algunas nociones pertenecientes á la Lógica y Metafísica» que enseñan el "modo con que conocemos los obgetos que nos presenta la naturaleza». Empieza con tono cartesiano a explicar el sentido de analizar para llegar a alcanzar ideas "claras y distintas» que se puedan dar a entender a otros. Sigue con el proceso de alcanzar ideas «universales ó abstractas» que «en las Matemáticas tienen mucho uso» y enuncia reglas para la sólida instrucción respecto a la verdad de los juicios, lo que le lleva a considerar las proposiciones (verdaderas o falsas, condicionales o absolutas) y sus demostraciones (directas o indirectas, como "el método de exâucion usado por los antiguos Geómetras»). La verdad de una proposición puede ser «evidente, cierta ó probable», pero el autor indica que las evidentes y las ciertas «pertenecen propiamente á las Ciencias , y las probables solo se estiman en quanto dirigen al conocimiento de la verdad á falta de las evidentes». Pasa después a considerar que un tratado científico expone proposiciones ciertas "con un orden natural" a partir de "primeros principios" evidentes, añadiendo que "segun varien los primeros principios de donde deriven los Autores los demas y sus consecuencias , variará tambien el orden ó sistéma de la doctrina». A continuación explica los términos: "Difinicion , Hipotesis , Observacion, Experimento , Experiencia, Axî̀ma , Postulado, Teorema, Problema, Corolario , Lema , y Escolio".

En la última parte de este segundo texto preliminar, el autor se dedica a "decir algo acerca de la utilidad de estas Ciencias ; del estudio que le convenga á

41. Rosell Viciano (1785), op. cit., pp. XXV-XLII. 
cada uno segun su carrera ; y de las disposiciones que se requieren para hacer el mayor progreso en dichas Ciencias». Respecto a la primera cuestión se expresa así:

[...] el estudio de las Matemáticas engendra mas que ningun otro amor á la verdad, aficion a lo sólido, y el método en todas las cosas ; y [...] para mantener el camino de la verdad en quanto pende de la razon. Estas luces, y las prácticas necesarias para el adelantamiento de las Artes, y facilidad del Comercio que suministra á cada paso con los felices descubrimientos á que dirige, muestran claramente quan importante es su estudio para el bien de la sociedad.

Afirma que instruirse en la aritmética universal y la geometría conviene a todos los que saben leer y escribir y tienen un "mediano talento". Luego se extiende sobre las múltiples profesiones a las que conviene una formación más avanzada en matemáticas. Y termina:

Finalmente, los que emprendan el estudio de las Matemáticas con ánimo de profesarlas , y los que se hallen empleados en destinos, cuyo desempeño dependa de la inteligencia en ellas, no solo deben estudiar todos los tratados con la mayor formalidad, sino que deben continuar despues con la lectura de la historia de estas Ciencias, y de las obras originales, hasta que habilitados en esto puedan gobernarse por sí , y elegir para su cultivo aquel ramo que mas convenga á su genio y destino, y al bien del estado.

Advierte después que el estudio de las matemáticas no se puede «tomar por diversion , [...] porque pide toda la atencion y aplicacion, tanto ó mas que ningún otro», y señala tres "disposiciones que se requieren para poder sobresalir en estas Ciencias»: la «inteligencia de lenguas» (recomienda la latina, la francesa y la inglesa) la edad para iniciar el estudio de las matemáticas (entre los 14 y los 20 años), y, la principal, el «talento y circunstancias de los principiantes: quanto mas dotados estén de aplicacion, constancia en mantener la razon, docilidad en ceder á ella, de consecuencia en sus razonamientos, y de imaginacion y penetracion, tanto mas se puede esperar superen las dificultades que se ofrecen en dicho estudio, y se aventajen en él». Los maestros, padres y tutores deberán observar estas disposiciones en quienes se inician en las matemáticas , "para no emplear inutilmente el tiempo y caudales, si se advierte que son mas aptos para otra profesión». Indica que debe seguirse un método en el estudio , dando hasta once reglas "que conviene observen los principiantes en el de estas Instituciones" ${ }^{42}$ , y que esos principiantes adelantarán mejor si «aprenden baxo la direccion de algun Maestro» que les ayude pero que «les oblígue á discurrir por sí, hasta poder continuar facilmente sin su ayuda; pues la sobrada explicacion engendra mas la creencia que la ciencia ; y dexa siempre á los discipulos en clase de principiantes».

42. En Dorce Polo, Carlos. Historia de las matemáticas en España. 2 vols. Prólogo de Luis Español. San Cugat: Editorial Arpegio, 2017, pp. 196-202 , están reproducidas las 11 reglas para el estudio; luego incluye un avance del contenido doctrinal de Instituciones. 


\subsubsection{Indice y organización}

Instituciones Matemáticas trata "de los Principios de la Aritmética universal,, con una primera parte, De la Aritmética numérica (caps. I-VI, pp. 5-188), y una segunda, De los principios de la Algebra (caps. I-IV, pp. 189-392). La obra está dividida en apartados de muy diversa extensión numerados del $§ 1$ al $§ 572$, que le sirven para las numerosas referencias internas que resaltan la ilación lógica y facilitan el estudio. Ofrece diversos enunciados que indica con abreviaturas en mayúscula y numeradas en arábigo. Los que llevan una numeración correlativa a lo largo de toda la obra son 5 axiomas ("AXÎ") al inicio de la obra, seguidos de un único postulado ("POSTUL»); 55 definiciones ("DIFIN") y 17 hipótesis ("HIPOT»), que se refieren en general a notaciones y 8 teoremas ( (TEOREM»). En realidad, hay bastantes teoremas más, porque muchos de los muy abundantes corolarios ( COROL») son como teoremas por incluir demostración, como reconoce el propio autor. Los corolarios van numerados correlativamente tras del enunciado principal al que acompañan, que a menudo es una definición o una hipótesis. Otro rasgo notable es que los párrafos explicativos van señalados poniendo una coma al inicio de cada una de sus líneas, de modo que si un lector quiere ceñirse al hilo principal del discurso teórico puede saltarlos. El estilo expositivo de la obra está marcado también por los 84 problemas que contiene, resaltados con toda la palabra en mayúsculas y colocada centrada en una línea seguida del ordinal correspondiente; luego, en la línea siguiente, viene el enunciado en cursiva precedido por un ordinal de apartado. Unos se refieren a cálculos y otros son teóricos. Los hay que ocupan varias páginas y contienen más de un apartado, con una o varias definiciones en su interior y frecuentes explicaciones adicionales. Los problemas presentan una resolución ( $(R E S »)$ y una demostración ( $(\mathrm{DEM}$ ), al estilo de las construcciones geométricas.

\subsection{Contenido doctrinal}

Las 392 páginas de contenido matemático empiezan con una "Introduccion» de corte enciclopedista. Partiendo de la observación de la naturaleza y de las nociones de extensión, relación, número y cantidad, define las matemáticas como "las Ciencias que tratan de las relaciones de la cantidad", siendo la cantidad "lo que es capaz de aumento ó disminucion». Luego establece su división en puras y mixtas y describe las subdivisiones de cada una de estas partes, siguiendo el esquema de la Encyclopédie ${ }^{43}$.

43. Véase D'AlemberT, Jean le Rond. Discurso preliminar de la Enciclopedia. Traducido por Consuelo Berges Rábago. Madrid: Sarpe, 1984, o bien Novy, Lubos. «Las matemáticas en la enciclopedia de Diderot y d'Alembert». Llull, Revista de la SEHCYT, 1993, 16, 30, pp. 265-284. 
Parte I. Axiomas, definiciones y postulados ( $\S \S 1-35$, pp. 5-14)

El capítulo primero ( $\$ \$ 1-68$, pp. 5-26) tiene un arranque conceptual que refiere al espíritu de la obra en su conjunto, por lo que vamos a comentarlo separadamente; la descripción del capítulo vendrá después.

El capítulo empieza con cinco axiomas y un postulado sobre la cantidad $^{44}$ :

AXÎO. 1. Quando á una cantidad no se le añade ó quita algo, queda la misma.

AXÎO. 2. La cantidad que es igual á alguna otra, es tambien igual á las iguales de ésta.

AXÎO. 3. La cantidad mayor ó menor que alguna otra , es tambien mayor ó menor que sus iguales.

AXÎO. 4. Quando en cantidades iguales se hacen las mismas operaciones con iguales , las que resultan de ellas serán tambien iguales.

AXÎO. 5. Quando en cantidades desiguales se hacen las mismas operaciones con iguales, las que resultan son tambien desiguales; esto es, las de la mayor mayores , y las de la menor menores.

POSTUL. Por cualquiera cantidad se puede substituir la que se quiera de sus iguales sin alteracion de aquella á quien se refiera.

Los axiomas y el postulado están explicados con ejemplos concretos sobre varas, pesos, onzas o árboles, en los que usa los enteros y los quebrados con su sentido intuitivo. Este inicio recuerda a los Elementos de Euclides, los axiomas son análogos a las nociones comunes ${ }^{45}$. La diferencia entre el postulado y el axioma 4 es el cambio de "operaciones» por "sustituciones", que se han de realizar en expresiones o fórmulas con cantidades previamente dadas usando el axioma 4. El lector de hoy echa en falta la versión simbólica de estos enunciados, porque Rosell ha resaltado en el prólogo el lenguaje simbólico como distintivo de su plan de traba$j^{46}$. No obstante, también opinó que las matemáticas se hacen con lenguaje verbal y simbólico, y que los símbolos se han de introducir paulatinamente.

Después de «estas verdades experimentadas por todos», Rosell pasa (pp. 7-12, $\$ \S 9-35)$ a definir las relaciones entre las cantidades. Lo hace mediante nueve definiciones, cada una de ellas seguida de varios corolarios que las aclaran o completan. La primera (\$9 DIFIN. 1) aborda el concepto de «relacion ó razon geométrica», que es aquella «en que se atiende á las veces que una cantidad contiene ó está contenida en otra». Siguen a esta definición dos corolarios, uno que aclara que

44. Cuando define en la Instrucción Proemial axioma y postulado (también llamado "peticion") los declara prácticamente sinónimos: verdades que no necesitan ser demostradas.

45. Las exposiciones más o menos fidedignas de los Elementos de Euclides del siglo XVIII llamaban con frecuencia axiomas a las nociones comunes. Así lo hace Tosca y quienes en él se inspiraron.

46. Denotando cantidades con letras minúsculas negritas y con los símbolos habituales, los axiomas dicen que las cantidades admiten expresiones como $\mathbf{p}=\mathbf{q}, \mathbf{p}<\mathbf{q}, \mathbf{p}+\mathbf{q}$, $(\mathbf{p}+\mathbf{q})+\mathbf{r}$, etc. El axioma 4 afirma que si $\mathbf{p}=\mathbf{q}$ y $\mathbf{r}=\mathbf{s}$ entonces $\mathbf{p}+\mathbf{r}=\mathbf{q}+\mathbf{s}$. Concluir de $\mathbf{p}+\mathbf{q}$ y $\mathbf{q}=\mathbf{r}+\mathbf{s}$ que $\mathbf{p}+\mathbf{q}$ $=\mathbf{p}+(\mathbf{r}+\mathbf{s})$ sería una instancia del postulado. 
es necesario que las cantidades a relacionar sean "de la misma especie» (para que se pueda decir que una contiene a otra) y otro que completa la definición inicial: "la razon geométrica se dirige tambien á representar la magnitud de la cantidad que se compare por medio de aquella con quien se compare. Rosell define ( $\$ 13$ DIFIN. 3) "unidad" como el consecuente de una relación, así que una unidad es una cantidad, señalando que "puede haber tantas unidades concretas en cada especie, como cantidades se puedan comparar en cada una de ellas". Por ejemplo, dice, hay muchas unidades de longitud. En general, una unidad es concreta de una determinada especie, pero hay también una "unidad abstracta» con la que se pueden comparar todas las cantidades independientemente de su especie. Luego (§ 16 DIFIN. 4), llama "número" a "la relación de qualquiera cantidad con la que se tome por unidad", se trata así de "números concretos" asociados a cantidades de cierta especie. Los "números abstractos" aparecen (§ 19 DIFIN. 6) «quando la cantidad se refiere á la unidad abstracta». Así, "tres y medio es abstracto , y tres varas y media concreto». Hasta aquí sus definiciones generales de relación y número, no mal orientadas, pero ciertamente imprecisas.

Para dar paso a la aritmética, más adelante afirma (\$ 30 DIFIN. 9) que el número es "entero" cuando "consta solamente de unidades", es "quebrado propio ó fraccion" si equivale a "parte" de la unidad y "quebrado impropio» si es "partes» de la unidad y mayor que ella. Añade que "la relacion que expresa el número entero es la mas simple de todas , y la que representa el quebrado es la de un entero á otro", de modo que "se debe mirar el número entero como el origen de los otros".

Parte I. Cap. I. "De la numeracion y arte de expresar los números." (\$§ 36-67, pp. 14-26)

Rosell entra en la aritmética ordinaria planteando las dos primeras «hipótesis» referidas a la expresión verbal y simbólica de los números enteros (solo positivos) en el sistema de numeración arábigo decimal, explicando la manera de escribirlos y leerlos con destreza pedagógica apoyada en un cuadro explicativo desde las unidades hasta los miles de trillones.

La tercera hipótesis ( $\$ 43$, p. 17) introduce la notación de letras para los «números indeterminados abstractos ó concretos" y la cuarta (§ 47, p. 19) la notación fraccionaria para las relaciones, ya sean numéricas determinadas, como 14/2, o indeterminada, como $a / b$. En el primer corolario explicativo de esta hipótesis, Rosell afirma que en una "relacion indicada $\frac{a}{b}$ " puede ser "que $a$ represente otra razon indicada $\frac{c}{d}$ y $b$ otra $\frac{e}{f}$, con lo que «la razon $\frac{a}{b}$ se podrá indicar tambien asi $\frac{\frac{c}{d}}{e}$ $(\S 7)$, donde la referencia interna envía al postulado sobre «sustituciones»" ${ }^{47}$. Indica

47. Rosell siempre escribe las relaciones con la raya horizontal, pero nosotros usaremos a veces la inclinada para mantener la escritura en línea si no hay confusión. Nunca se considera que el denominador sea cero. 
también que lo mismo significa $b$ que b/1. En la hipótesis sexta (§ 61, p. 23) Rosell introduce la notación para los quebrados con denominador el uno seguido de ceros y también la manera de leer estos números decimales, incluyendo otro cuadro explicativo.

Parte I. Cap. II. "De la composicion y resolucion de los números en general." (\$§ 69-134, pp. 26-45)

En este capítulo, Rosell define las operaciones «sumar, multiplicar, y elevar un número á qualquiera dignidad». Para empezar: "70 DIFIN. 11. Sumar es juntar dos ó mas números homogéneos dados, y expresarlos por uno solo. A este número llamamos suma ó agregado, y á los dados sumandos". Explica en corolarios que la suma es de la misma especie que los sumandos, pero enseguida se refiere a los enteros sin especie: un entero es suma de unidades y la suma de números enteros es otro entero.

Luego define la multiplicación como suma repetida del multiplicando las veces que indica el multiplicador, advirtiendo que el multiplicando puede ser un número concreto pero el multiplicador debe ser abstracto: "Algunas veces se propone multiplicar un número concreto por otro, como si se pidiera el valor de 4 varas de lienzo á razon de 6 reales cada una, en cuyo caso parece se debian multiplicar los 6 reales por 4 varas, pero en la realidad no se multiplica 6 reales sino por 4 tomado abstractamente ( $\$ 23)$ ); una referencia interna lleva al $\S 75$ en el que había explicado que cada número concreto presenta "las mismas relaciones» que el abstracto que resulta de prescindir de su especie. Si no consta especie, la multiplicación de enteros es un entero y no importa el orden en que se tome, propiedad que explica mediante un eficaz diagrama centrado en un rectángulo de unidades. Aborda una propiedad conmutativa más general que se presenta cuando el multiplicando es un número entero de veces una cantidad, pero le parece suficiente ilustrarla con un ejemplo: «Si se han de multiplicar 6 pies por 4 deberá resultar el mismo producto multiplicando 4 pies por 6». Deja la explicación a la inspección de un diagrama como el usado con los enteros, pero donde allí ponía 1 ahora pone "1p" $(1 \mathrm{pie})^{48}$. La potencia tiene un tratamiento muy breve. Como es el producto de un número por sí mismo, el número tiene que ser abstracto.

48. De momento, Rosell solo opera con enteros y no ha iniciado el uso del lenguaje simbólico. Con símbolos, si $a$ es un número entero y $\boldsymbol{p}$ una cantidad, tenemos el número concreto $a \boldsymbol{p}$ que suma $\boldsymbol{p}$ consigo misma $a$ veces. Rosell afirma que $(\mathrm{ap}) \cdot \mathrm{b}=(\mathrm{b} \mathbf{p}) \cdot \mathrm{a}$, y la prueba que el diagrama propuesto sugiere es que $(\mathrm{ap}) \cdot \mathrm{b}=(\mathrm{ab}) \mathbf{p},(\mathrm{b} \mathbf{p}) \cdot \mathrm{a}=(\mathrm{ba}) \mathbf{p}$, y la igualdad propuesta sigue de la conmutatividad de los enteros abstractos. 


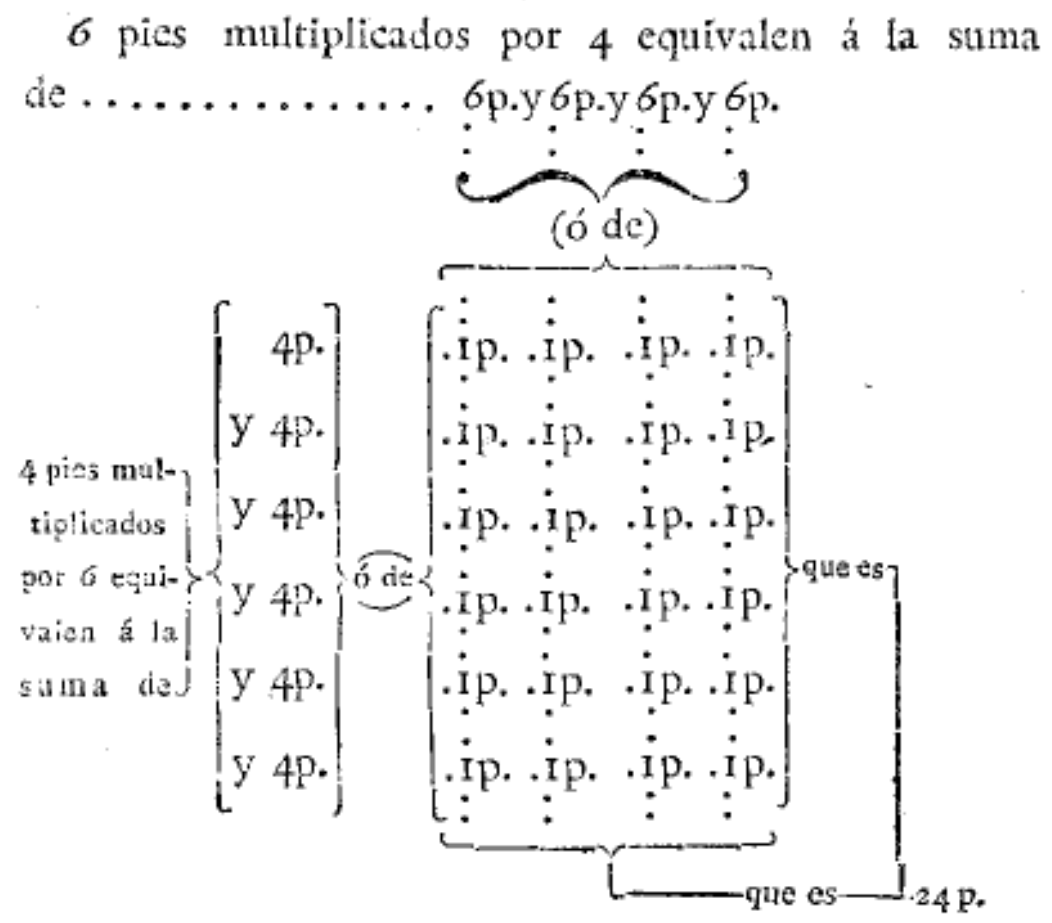

Fig. 3. Conmutatividad en la multiplicación.

Fuente: Rosell, 1785

A continuación, Rosell pasa a ver que las tres propiedades anteriores tienen sus «Contrarias [...] Restar, Dividir y Extraher la raiz». "§ 88 DIFIN. 14. Restar es hallar lo que queda de un número quitándole otro homogéneo», de modo que la resta es lo que queda, es decir, el "Subtrahendo" más la "resta» es el "minuendo". Aparece el símbolo 0 como resta de números iguales, y si el sustraendo es mayor, "no se podrá efectuar la subtraccion", pero "la resta será lo que le falte al minuendo para poder executar la subtraccion, ó lo que le quedará por restan.

Afirma (p. 34, § 93 DIFIN. 15) que «dividir ó partir es hallar las veces ó modo con que un número contiene á otro" (ambos homogéneos) o, lo que es lo mismo, "hallar la relacion de un número con otro", por lo que unifica la notación de relaciones y de quebrados, resultando que $a / a$ es 1 y que «el producto del divisor por el quociente debe ser igual al dividendo", es decir, multiplicando $b$ por $a / b$ resulta $a$, o bien multiplicando $c / d$ por $(a / b) /(c / d)$ resulta $a / b$. Afirma que el cociente $(a / b) / a$ es igual a $b$, siendo $a, b$ enteros o quebrados y prueba que $a$ por $b / c$ es 
igual a $b / c$ por $a$ : por la propiedad anterior, $a$ por $b / c$ dividido por $a$ es $b / c$ y se aplica que cociente por divisor es igual a dividendo. A continuación, Rosell inicia ( $\$ 112$, p. 38) el estudio de la divisibilidad considerando los cocientes exactos, que indican que el divisor es «medida" del dividendo, terminología que viene de Euclides. Si $b$ es medida de $a$, también $a / b$ es medida de $a$. Define los números primos, que llama «simples» o "primeros» y expone la descomposición de todo número en factores primos sin mencionar la unicidad. Tras la división, sigue (p. 40, $\S 123$ DIFIN. 20) la extracción de raíces (cuadradas, cúbicas, etc.) mencionada de un modo simplemente nominal, introduciendo definiciones.

Terminada la exposición inicial de las seis operaciones cuyos aspectos prácticos se verán en el capítulo siguiente, el autor introduce notaciones a través de las hipótesis 7 y 8 y sus respectivos corolarios. Este es el momento que le parece oportuno para desplegar el lenguaje simbólico añadiendo a las letras ya usadas los signos matemáticos de uso ordinario: igual, mayor, menor, más, menos, producto (el aspa y el punto), la división como dos puntos y las raíces, con «\&c" como símbolo para etc. Como ejemplos del uso de los signos repite en forma simbólica enunciados que habían aparecido antes en forma verbal. En cuanto a resta y negativos, explica que la expresión $-a$ denota que la cantidad $a$ se debe restar y escribe $a-a=0$. Suponiendo $a>b$, escribe la diferencia $a-b=c$, indicando que también se tiene $b-a=-c$; en efecto, la primera diferencia indica que $c$ se debe sumar a $b$ para obtener $a$ y la segunda que $c$ se debe restar a $a$ para obtener $b$. Con el producto vemos expresiones como $1 a=a 1=a, 0 a=a 0=0, a+a+a+\& c=m a$, siendo $m$ el número de repeticiones, $a b+a b=2 a b, a b c d=a c b d=b c a d=c b a d=\& c$. Con la división propone $a b c d e / a c d=$ be y otras análogas permutando las letras.

Parte I. Cap. III. "De la adicion, subtraccion, multiplicación, division, y medidas de los números enteros abstractos.» (\$\$ 135-168, pp. 45-93)

Rosell vuelve a tratar de las operaciones, pero con los algoritmos de cálculo en el sistema de numeración decimal. Organiza el capítulo en problemas, los cuatro primeros uno para cada una de las cuatro operaciones. La exposición es pedagógica, atenta a los detalles, con tablas y cuadros, presentando casos de creciente dificultad y con suficientes ejemplos.

Halla los factores primos («primeros») de un número entero por el método de criba, indicando que ha puesto al final del libro una tabla con las factorizaciones primas de los números hasta 2000. Halla los divisores ("medidas») de un número organizando todos los productos posibles con sus factores primos contando bien las repeticiones. Da ejemplos en unos cuadros ilustrativos, pero no la fórmula del número de divisores. Aborda el cálculo del mínimo común múltiplo y del máximo común divisor de dos números a través de la factorización prima y para el segundo enseña también el algoritmo de las divisiones sucesivas, haciendo notar que este último "se debe preferir al otro quando los números dados son muy crecidos". También usa la factorización prima para probar: «§ 167. COROL. 2. Si un entero no 
mide á otro tampoco medirá qualquiera potencia del primero á la misma potencia del segundo".

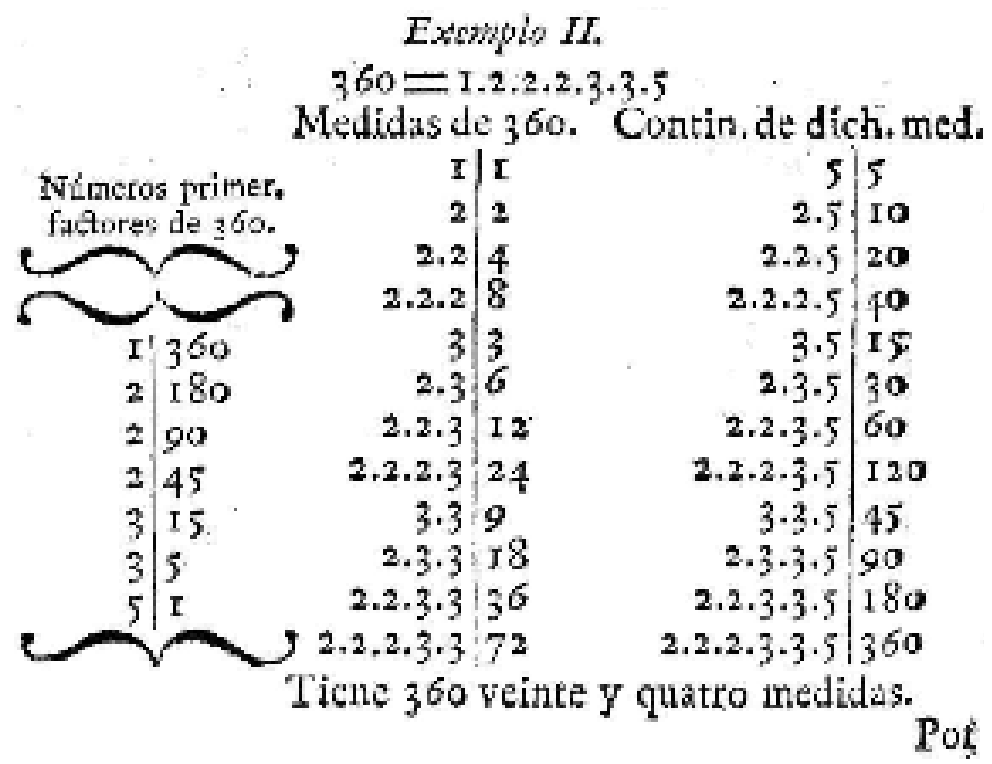

Fig. 4. Factores primos y divisores de 360.

Fuente: Rosell, 1785.

Antes de pasar al capítulo siguiente, el autor deja planteados unos ejercicios (I a VI) de cálculo con divisiones y divisores, porque «importa mucho que los principiantes se exerciten en la práctica de todas las operaciones». En casi todos los capítulos que siguen, repite esta misma recomendación antes de dejar planteados unos cuantos ejercicios de cálculo para resolver; este hecho ya no lo volveremos a mencionar en lo sucesivo.

Parte I. Cap. IV. "De la adicion, subtraccion, multiplicacion, division, y reduccion de los números abstractos, mixtos y quebrados.» (\$\$ 169-231, pp. 94-130)

En este capítulo, Rosell enseña a operar con quebrados (problemas 9 al 20). No nos fijaremos en las operaciones con los números mixtos, que ocupan cierta extensión al uso de la época, pero simplemente se reducen a aplicar lo dicho para enteros y quebrados. Comienza por la suma y resta de quebrados con igual denominador, sigue el producto de un entero por un quebrado, lo que aprovecha para dar la expresión $a / b=a \times 1 / b$, que le permite realizar cálculos como $(a / b) / c$ $=(a / b) \times(1 / c)=a 1 / b c=a / b c$. Luego convierte cada entero en un quebrado con denominador dado y multiplica dos quebrados. 
En este punto aparece el primer enunciado que Rosell rotula como teorema (p. 106, § 193 TEOREM. 1), en el que vincula el producto con la igualdad y el orden. Para abreviar la explicación de este teorema y sus dos primeros corolarios, escribiremos los quebrados con una sola letra: el teorema afirma que $a \cdot b=a$ si el numerador y el denominador de $b$ son iguales $(b=1)$ y lo mismo cambiando igual por menor o por mayor. Como corolarios obtiene que si $a<1$ entonces $a^{2}<$ $a$ y el análogo con mayor ${ }^{49}$. Llega después el segundo teorema (p. 108, § 196 TEOREM. 2), que afirma que un quebrado no cambia si se multiplican su numerador y su denominador por el mismo entero; lo demuestra poniendo $a c / b c=(a / b)(c / c)$ $=(a / b) 1=a / b$. En los corolarios de este teorema trae a colación quebrados con numeradores y denominadores terminados en varios ceros y pasa a considerar las operaciones con los números decimales. También aborda la reducción de quebrados a común denominador tomando el producto de los denominadores, con lo cual ya puede comparar, sumar y restar quebrados cualesquiera. Para la división de quebrados primero propone la fórmula $(a / b):(c / d)=(a d / b c)$ y luego demuestra que es correcta usando el teorema 2. Simplifica quebrados usando el máximo común divisor y convierte un quebrado en otro con denominador dado (cuarta proporcional) de nuevo por el método de resolución y demostración: dado $a / b$ y un denominador $d$ propone $(a d / b) / d$ y prueba que es la solución correcta usando reglas operativas ya demostradas. Utiliza este resultado para aproximar por defecto un quebrado $a / b$ con error menor que $1 / d$, lo que muestra con ejemplos, sin formulación general. Para expresar las fracciones como números decimales ${ }^{50}$ calcula, por ejemplo, $\frac{6}{7}=0,8 \frac{4}{7}=0,85 \frac{5}{7}=0,857 \frac{1}{7}$; "suprimiendo los quebrados de los numeradores» obtiene las que llama "aproxîmantes" decimales sucesivas ${ }^{51}$.

Parte I. Cap. V. "De la composicion y resolucion de las potencias segundas y terceras de los números abstractos.» (\$§ 232-258, pp. 131-154)

Aquí Rosell enseña a extraer las raíces cuadrada y cúbica (problemas 21 a 23). Expone primero el algoritmo de la raíz cuadrada a partir del desarrollo de $a+b$ al cuadrado, " $a a+2 a b+b b »$, dando ejemplos dispuestos en cuadros muy ilustrativos. La exposición similar del algoritmo de las raíces cúbicas parte del cubo de $a+b$ (pp. 146-154).

Como corolario del algoritmo de la raíz cuadrada ofrece ( $\$ 242$, p. 142) un importante resultado teórico: "si no se halla la raiz exâcta de un número entero en enteros , tampoco se ballará en quebrados ni mixtos", lo que le lleva a considerar

49. Usará estos corolarios y el ya comentado $\S 167$ para demostrar en el capítulo siguiente (§ 242) que $\sqrt{n}$ no es un quebrado si $n$ no es un entero cuadrado perfecto.

50. Al considerar la expresión decimal de los quebrados nunca menciona la posible existencia de periodos.

51. El autor se refiere a "quebrados de los numeradores" porque entiende que $0,8 \frac{4}{7}=\frac{8+\frac{4}{7}}{10}$, etc. Así que la expresión que ha dado es $\frac{6}{7}=0,8+\frac{4}{70}=0,85+\frac{5}{700}=0,857+\frac{1}{7000}$ 
nuevos números «irracionales, inconmensurables, sordos , ó inexplicables» (\$ 243, p. 142 , DIFIN. 23), como $~ \sqrt{ } 2, \sqrt{3}, \&$ c.» ${ }^{52}$.

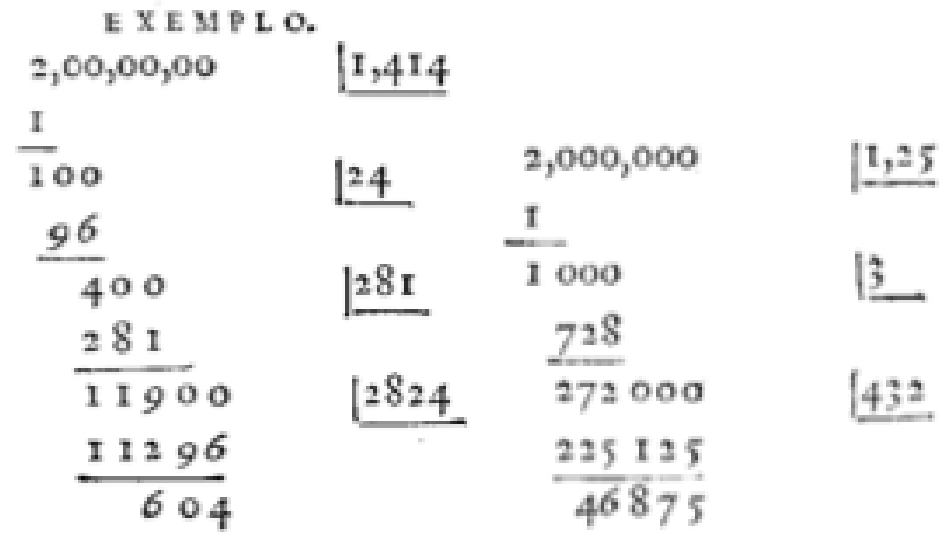

Fig. 5. Aproximaciones de $\sqrt{2}$ y $\sqrt[3]{2}$.

Fuente: Rosell, 1785

Para las raíces de los quebrados da la fórmula $\sqrt{\frac{a}{b}}=\frac{\sqrt{a}}{\sqrt{b}}=\frac{\sqrt{a b}}{b}$, que usa para aproximar: si $c$ es la aproximación por defecto que el algoritmo da para $a b$, entonces $c / b$ es una aproximación por defecto de $\sqrt{a / b}$, y la aproximación mejorará cuanto más grande se tome $b$. Calcula las raíces de números decimales asimilándolos a fracciones, y con ello aproxima las raíces cuadradas que no son exactas.

Parte I. Cap. VI. "De la composicion y resolucion de los números concretos." (\$\$ 259-290, pp. 155-188)

El objetivo de este capítulo (problemas 24 a 38) es calcular con números "Complexôs» expresados en unidades homogéneas de diverso tamaño y uso popular, por ejemplo, 4 varas 2 pies y 7 pulgadas. Comienza Rosell con una tabla de las diversas unidades en uso en el país, sus nombres y abreviaturas, y, entre las homogéneas, los factores de conversión de unas a otras; las hay de dinero, peso, tiempo y longitud. En otras tablas da la correspondencia del pie castellano con "medidas estrangeras antiguas, y modernas", en especial de Francia. El capítulo presenta una gran variedad de ejemplos con cálculos concretos, pero ofrece también un esquema teórico que empieza ( $\$ 265$, p. 158) considerando unidades de tamaño decreciente que denota $A, B, C, D, E, \& c$., y números "complexôs» $m A+$ $n B+p C+q D+r E+\& c$. Las conversiones sucesivas son $A=a B, B=b C, C=c D$,

52. El algoritmo proporciona aproximaciones sucesivas de estos números y Rosell remite a la geometría del segundo tomo para que se aprecie su existencia como «expresion del valor de algunas lineas». 
LUIS ESPAÑOL GONZÁLEZ Y JUNCAL MANTEROLA ZABALA

$D=d E, \& c$. Para "reducir qualquier número complexô á quebrado impropio de la especie superion obtiene la fórmula

$$
m A+n B+p C+q D+r E=\frac{m a b c d A+n b c d A+p c d A+q d A+r A}{a b c d}
$$

Parte II. Cap. I. "De algunas nociones y operaciones fundamentales del Algebra propiamente dicha.» (\$\$ 291-383, pp. 189-236)

Empieza Rosell con las definiciones de ecuación, fórmula, término, monomio, polinomio, coeficiente, etc., señalando que algunas expresiones con letras ya han sido usadas en la primera parte. Por polinomio entiende cualquier expresión con sumas y diferencias de productos de números y letras, donde los números son los coeficientes; los monomios son los términos que van sumados o restados en los polinomios. Por ecuación entiende cualquier igualdad entre polinomios, sin letras especiales que designen incógnitas.

En el siglo XVIII, el álgebra era el lugar para introducir los negativos como recurso operacional, así que el primer resultado que enuncia Rosell es (p. 192): « 298 TEOREM. 3. Los signos + y - que preceden a las cantidades de una misma expresion manifiestan que estas concurren en sentidos opuestos al fin del cálculo" ${ }^{53}$. Luego define que una cantidad sin signo es positiva, al igual que si aparece con signo +, y es negativa si va precedida del signo -. En las explicaciones de esta cuestión aparecen múltiples ejemplos en los que el signo va asociado a la especie (longitud, tiempo, dinero, etc.) y no a la cantidad abstracta, que siempre es positiva.

Al iniciar la simplificación de las expresiones introduce la notación exponencial para las potencias (p. 196, § 304, HIPOT. 11), lo que le permite completar lo dicho sobre esta operación en la parte primera. Simplifica expresiones como, " $3 a b \times 2 a c=6 a^{2} b c$ y da reglas operativas: " $a^{m} \cdot a^{n}=a^{m+n}$ y " $\left(a^{m}\right)^{n}=a^{m n}$, también $《 \frac{a^{m}}{a^{n}}=a^{m-n}$. Introduce la notación exponencial para las raíces, $\sqrt[4]{a^{n}}=a^{\frac{n}{m}}$, con lo que genera un cálculo formal con radicales, independientemente de que la raíz sea un número exacto o no, demostrando (p. 204, §325 TEOR. 4) que « $a \bar{m} »$ es irracional si la fracción del exponente no es un entero y el corolario: "Qualquiera cantidad irracional equivale á sí misma dividida por la unidad». Este es un resultado significativo para valorar la exactitud (lo que ahora llamamos rigor) que Rosell tiene en mente, pues ya ha demostrado lo anterior primero con enteros y luego con quebrados, ahora le toca con los irracionales que aparecieron en su camino, para él un irracional es un radical $\sqrt[\pi]{a^{n}}$. Luego da la regla $\sqrt[m]{\sqrt[n]{a}}=\sqrt[m n]{a}$ y concluye (p. 209, § 335 TEOR. 5) que las potencias con exponente fraccionario se operan igual

53. Le parece importante resaltarlo, pero ni es un teorema ni la larga explicación es una demostración. 
que las que lo tienen entero. Rosell hace notar que (p. 210, § 337 COROL. 2) «qualquiera cantidad racional equivale al producto del número de factores irracionales que se quiera». Después de siete corolarios más con ejemplos de cálculo con potencias fraccionarias, viene un nuevo resultado conceptual (p. 215, \$347 TEOR. 6): no importa el orden cuando se multiplican cantidades irracionales (radicales), algo que Rosell había probado ya con enteros y quebrados. El capítulo continúa con ejemplos de cálculo, que a partir de (p. 220, § 353 HIPOT. 13) expresan radicales no solo de letras sino de expresiones polinomiales. Llega un momento en que el autor introduce letras mayúsculas para representar polinomios, a los que llama también "cantidades», de modo que se pueda operar con ellas como si fueran las minúsculas antes usadas para representar números (pp. 226-228, TEOR. 7-8). Esta ampliación de las cantidades a las que aplicar el álgebra lo lleva a dar de nuevo las reglas de los signos para la suma, el producto y el cociente, estos últimos solo con monomios.

El paso siguiente en la exposición es considerar signos en las potencias, asunto breve, y en las raíces, lo que necesita más atención. Empieza observando que una raíz par solo puede obtenerse de una cantidad positiva, en cambio, una raíz impar puede obtenerse de una cantidad de cualquier signo, pero la raíz tendrá el mismo signo que la cantidad dada. Al afirmar (p. 231, § 368 COROL. 7) que «la raiz par de qualquiera cantidad positiva puede ser positiva ó negati$v a$ * comete una imprecisión verbal que se agrava cuando escribe $\sqrt[2 n]{a}= \pm \sqrt[2 n]{a}$, lo que le llevará a cometer errores más adelante. Rosell afirma que «no puede haber raices pares de las cantidades negativas", pero a continuación (p. 232, § 372 DIFIN. 32) admite que estas aparecen en los cálculos, una tal raíz es «imaginaria ó imposible, á diferencia de todas las demas, que se llaman reales». Establece que una raíz imaginaria es una expresión de la forma $" \sqrt[2 n]{-a}$, siendo $a$ una cantidad real (positiva). Aparece de inmediato la raíz cuadrada $\sqrt{-1}$ y con ella un primer cálculo: $(a \sqrt{-1})^{2 m}= \pm a^{2 m}$, positivo si $m$ par y negativo si impar; después con exponente $2 m+1$ obtiene el resultado $\pm a^{2 m+1} \sqrt{-1}$. A partir de aquí, durante las cuatro últimas páginas del capítulo, Rosell se muestra confuso y comete errores. Demuestra la fórmula $\sqrt{-a b}=\sqrt{a} \times \sqrt{-b}$ con un ć́rculo vicioso; luego, con $a=1$, deduce $\sqrt{-b}=1 \times \sqrt{-b}$, igualdad que amplía a raíces cuadradas imaginarias una propiedad de los enteros ya extendida con demostración formal a quebrados e irracionales radicales. Luego, presenta unas fórmulas con las que pretende poner de manifiesto que la multiplicación y división de las raíces imaginarias no son del todo iguales a las mismas con reales, y busca formular una regla de los signos específica para el caso imaginario. La primera fórmula es $\sqrt{-a} \times \sqrt{-b}=-\sqrt{a b}$, probada operando con $\sqrt{-a}=\sqrt{-1} \sqrt{a}=(-1)^{\frac{1}{2}} a^{\frac{1}{2}}$, método que podía haber usado en la fórmula anterior. Pero después ofrece otra prueba alternativa descabellada, usando $\sqrt{-1} \times \sqrt{-1}=\sqrt{-1 \times-1}=1$, un error grueso, o mejor absurdo. Con este mal proceder obtiene como resultado $\sqrt{a b}$, pero lo transforma en su opuesto 
argumentado que en la raíz cuadrada de un número positivo hay ambigüedad de signo y en este caso hay que tomar $\sqrt{a b}=-\sqrt{a b}$. Sin duda en este momento a Rosell se le fue la cabeza. Siguiendo con este desafortunado proceder, Rosell prueba fórmulas de cocientes análogas a la anterior: $\sqrt{a}: \sqrt{-b}=-\sqrt{-(a: b)}$, etc. El autor andaba confuso con el asunto de los dos valores de una raíz cuadrada de número positivo, confusión que mantiene con las raíces imaginarias, pues en el primer corolario de la definición 32 afirma que "á las raices imaginarias, aunque pares, no les corresponde ambiguedad de signo". Mayor es la desorientación cuando se atreve, al final, con raíces de grado par arbitrario, dando una fórmula desafortunada. En definitiva, igual que antes surgieron los irracionales limitados a radicales, ahora lo hacen los imaginarios como radicales pares de números negativos, pero Rosell no llega a comprender este nuevo tipo de cantidades.

Parte II. Cap. II. "De las demas operaciones del Algebra propiamente dicha." (\$§ 384-404, pp. 237-271)

Este es un nuevo capítulo de problemas (del 37 al 42), en los que se explica el modo de calcular con polinomios con un número cualquiera de letras, en general de las primeras del alfabeto, y un coeficiente numérico. A veces calcula también con quebrados de polinomios. Los dos primeros capítulos se refieren a sumas y restas, que se realizan por yuxtaposición con agrupamiento de términos semejantes. En el tercero se explica la multiplicación de polinomios mediante ejemplos y paso a paso, primero monomios por monomios, luego monomios por polinomios y finalmente el caso general. Dedica un problema a la factorización, ofreciendo solo ejemplos. Luego aparece descrito verbalmente el algoritmo de la división de polinomios ordenados respecto a una letra, que empieza a ser denotada $x$. Por ejemplo, calcula

$$
\frac{c x^{4}+b c x^{3}+6 c^{3} x^{2}+c^{4} x}{c x^{2}-b x^{2}-3 c^{2} x}=x^{2}-2 c x-b x+\frac{c^{4} x-b c^{3} x}{c x^{2}-b x^{2}-3 c^{2} x}
$$

En el «Exemplo VII», Rosell llena la p. 259 con el algoritmo de una división que explica en la página siguiente, cuyo cociente se puede extender indefinidamente:

$$
\frac{a^{2}}{b+c}=a^{2}\left(\frac{1}{b}-\frac{c}{b^{2}}+\frac{c^{2}}{b^{3}}-\frac{c^{3}}{b^{4}}+\frac{c^{4}}{b^{5}}-\& c\right)
$$

lo que le da pie a una nueva definición (p. 261):

399 DIFIN. 37. Una expresion de terminos sin fin se llama série, y esta se llama convergente, si sus terminos disminuyen succesivamente de valor ; divergente, si aumentan ; y es tanto mas convergente ó divergente quanto mayor sea la diferencia de un termino á otro. 
Justifica que la expresión del quebrado $\frac{1}{b+c}$, que se obtiene en la división ilimitada anterior haciendo $a=1$ es una serie divergente si $b<c$, convergente si $b$ $>c$ y "tanto mas convergente, quanto mayor sea $b$ respeto de $c{ }^{54}$.

En el último problema se aborda el cálculo del máximo común divisor de dos cantidades algebraicas esbozando el algoritmo de las divisiones sucesivas usado con los enteros. Ofrece un conjunto de explicaciones verbales acompañadas de algún ejemplo, no tantos como en otras partes del libro. Antes de cerrar el capítulo afirma que las potencias y las raíces de los polinomios las tratará «en otro lugar».

Parte II. Cap. III. "De várias propiedades de las relaciones de las cantidades en general.. (\$\$ 405-492, pp. 272-316)

El capítulo comienza recapitulando lo realizado en los anteriores en términos de operaciones con cantidades y relaciones, resaltando "de quan distintos modos puede resultar una misma relacion» mediante las «equaciones». Anuncia que dará a conocer nuevas formas de igualar relaciones necesarias "para lo que falta de la Aritmetica universal, y para la Geometría». La primera novedad aparece con las nociones de variable y límite:

422 DIFIN. 43. Quando una cantidad varía , acercandose á alguna otra cantidad ó término, de suerte, que jamás puede llegar a él, pero sí acercarse, de modo que la diferencia de ella al termino pueda ser menor que qualquiera cantidad por pequeña que sea, aquella cantidad ó termino, á quien se acerca se llama límite suyo.

El primer ejemplo es el quebrado $1 / 2$ y sus mitades sucesivas que «disminuirán continuamente de valor , y se acercarán mas y mas á no valer nada ó cero», así que su límite es "cero, ó 0/1." Luego escribe las expresiones $a-x$ y $a+x$ para indicar que si en $x$ se colocan las sucesivas mitades anteriores resultarán otras dos cantidades variables, ambas con límite $a$. El siguiente ejemplo es la serie obtenida en el capítulo anterior para $\frac{1}{b+c}$ en el caso convergente $b>c$, cuyos términos (positivos, sin contar el signo) decrecen con límite cero y las sumas de los términos (con signo) varían con límite $\frac{1}{b+c}$. Para las «cantidades ó razones que crecen sin fin ó al infinito» introduce como límite el símbolo $\infty=1 / 0$, con lo que resulta $0=1 / \infty$, pero, curándose en salud religiosa, advierte (p. 281) que

[...] el infinito de que hablan los Matemáticos no es el infinito verdadero, sino el límite al qual se acercan mas y mas las cantidades que crecen sin fin, sin poder llegar á igualarse con él, del mismo modo que las que decrecen se acercan á cero, sin poder llegar á ser cero.

54. Nótese que, aunque en $b+c$ ambos sumandos juegan un papel simétrico, el autor ha optado al dividir por expresar el cociente en desarrollo de potencias de $c$, como si $c$ fuera la variable y $b$ el coeficiente. 
Rosell compara los infinitos $1 / b$ y $(1 / b)^{m-1}$ cuando $b$ tiende a cero, llegando a conclusiones que simboliza así: $a \infty^{m} \pm c \infty^{m-1}=a \infty^{m}$ y $a: \infty^{m} \pm c: \infty^{m+1}=a: \infty^{m}$. Obtiene también $a \pm c: \infty=a$, que en particular le da $a \pm 0=a$, lo que no entiende como una operación entre cantidades, sino como una aplicación de su axioma 1. Para Rosell 0 e $\infty$ no son propiamente cantidades, una por ser "nada" y la otra por ser infinito, pero esto no contradice que puedan ser límite de cantidades variables, como explica en una nota al pie (p. 282), en la que también menciona que $1 / \infty$ se mira como "cantidad infinitamente pequeña en contraposicion de $\infty$, que se mira como infinitamente grande». En otra nota al pie (p. 286), destaca que los infinitesimales acarrean inexactitud y contradicciones que promovieron disputas entre los partidarios de Newton y los de Leibniz, con las que ha terminado D'Alembert mediante los límites, alternativa a las fluxiones y los infinitesimales. Después de estas consideraciones, Rosell da dos teoremas sobre límites:

434 TEOR. 9. Si a es limite de la cantidad variable $\mathrm{x}, y \mathrm{~A}$ de la cantidad variable $\mathrm{X}, y$ acercandose $\mathrm{x}$ á su límite a , y X á su limite $\mathrm{A}$, guardan siempre entre sí una misma relacion $[. .$.$] será la razon de los límites \frac{\mathbf{a}}{\mathbf{A}}$ igual á la de las cantidades $\frac{\mathbf{x}}{\mathbf{x}}$, de quienes son límites.

440 TEOR. 10. Si a es limite entre las dos cantidades variables $\mathrm{x} y \mathrm{z}, \mathrm{y}$ A entre otras dos variables $\mathrm{X} y \mathrm{Z}$; de suerte, que $\mathrm{x}<\mathrm{a}<\mathrm{Z}, y \mathrm{X}<\mathrm{A}<\mathrm{Z}, y$ que mientras las quatro

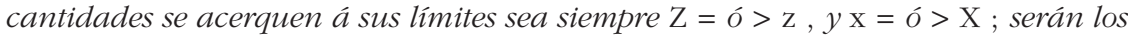
limites a $y$ A iguales.

La demostración del primero consiste en mostrar que a/A no puede ser ni mayor ni menor que $\mathrm{x} / \mathrm{X}$ y la del segundo de modo similar: las desigualdades $a>A, a<A$, son imposibles. El primer teorema le lleva a considerar la situación 0/0, que no es una relación entre cantidades sino un símbolo indicativo de límites. En el caso de las cantidades variables $a(c-x)$ y $b(c-x)$, encuentra que $0 / 0=a / b$, también da ejemplos con $0 / 0=0$ y con $0 / 0=\infty$. Remite al cálculo diferencial para encontrar «reglas mas faciles y generales para hallar el valor de las expresiones que se reducen á $\frac{0}{0}$ en todos los casos", pero antes de pasar a otro asunto menciona el modo de reducir a 0/0 las indeterminaciones $\infty / \infty, 0 \mathrm{x} \infty, \infty-\infty$.

Sin solución de continuidad, empieza (p. 293, § 441, DIFIN. 44) a exponer la teoría de las proporciones de Eudoxo-Euclides: una "proporción geométrica" es una igualdad de razones geométricas, $a / b=c / d$, o bien escrita en la forma $a: b::$ $c: d$; se llama "discreta" si todos sus términos son diferentes y "continua" cuando $b=c$, que es entonces el "medio proporcional». Explica, aportando ejemplos, que, aunque las razones se dan entre cantidades de la misma especie, las proporciones pueden igualar razones de especies distintas que han de «representar las dos la misma cantidad». Es muy significativo el corolario 3 de la definición anterior, porque en él se observa la evolución que Rosell pretende respecto a los Elementos de Euclides. En dicho corolario, demuestra que en toda proporción $a / b=c / d$, el producto de los medios es igual al producto de los extremos: $a d=b c$. Lo prueba multiplicando los dos miembros de la proporción por $b d$ y simplificando. Luego 
aclara que las multiplicaciones se harán «segun se previno en § 75», aclaración pertinente porque no es posible, por ejemplo, multiplicar 7 varas por 6 pies, pero en el apartado citado explicó que al multiplicar dos cantidades concretas la segunda se toma como abstracta; así justifica que los productos efectuados son consistentes. Rosell ha formulado su teoría de las proporciones (con escasa fortuna en cuanto al pretendido rigor expositivo similar al griego) de modo que puede hacer los productos cruzados en modo algebraico ${ }^{55}$. Con el corolario 3 y su inverso el corolario 7 demuestra de modo algebraico sencillo, en corolarios sucesivos, las propiedades comunes de las proporciones ${ }^{56}$.

Termina la teoría de las proporciones geométricas demostrando que los productos y las potencias, las divisiones y las raíces conservan la proporcionalidad.

A continuación (pp. 302, § 461 DIFIN. 45) considera la noción de «razon aritmetica" de dos cantidades, que es una diferencia $a-b$ en vez del cociente en el caso geométrico. Si $d$ es la diferencia (el mayor menos el menor) de los números $a, b$, entonces la razón aritmética $a-b$ es igual a $\pm d$ y también a $a-(a- \pm d)$. Rosell ofrece en este punto ( $(464)$ una imagen interesante de los enteros de ambos signos todos ellos ordenados. Como tiene $0-(-c)=c$ con $a>b$ y $a-b=$ $c$, será $0>-c$, así que "cero es mayor que una cantidad negativa". De un modo análogo prueba que «una cantidad negativa menor es mayor que otra menon, trabalenguas que queda más claro en su versión simbólica: $-a>-c-a^{57}$. También demuestra que "una cantidad positiva es mayor que una negativa». Después de las razones aritméticas llegan las igualdades entre ellas, que son (pp. 303, §465 DIFIN. 46) las "proporciones aritméticas" $a-b=c-d$; se trata de la noción correlativa de las proporciones geométricas, cambiando división por resta y producto por suma. Con estas nociones, el autor repite parte de lo dicho para las geométricas.

Tres cantidades son continuamente proporcionales si $a / b=b / c\left(a c=b^{2}\right)$ en el caso geométrico y si $a-b=b-c(a+c=2 b)$ en el caso aritmético. Al modo griego, Rosell utiliza la proporcionalidad continua para definir (p. 305, § 471 DIFIN. 47) una "progresion" como una "serie de cantidades continuamente proporcionales», que será "aritmética» o "geométrica» según el tipo de proporcionalidad que se utilice. Luego, desgrana las propiedades básicas de unas y otras de modo simultáneo y correlativo.

Rosell observa que, empezando por la unidad, las potencias sucesivas de una cantidad forman una progresión geométrica y arranca con los logaritmos,

55. Cuando Euclides enuncia esto en Elementos no lo hace en el libro V de las proporciones, independiente de los libros geométricos anteriores, sino en el libro VI de la semejanza, donde (proposición VI6) toma una proporción entre segmentos para que los productos cruzados den una igualdad entre rectángulos, no entre segmentos.

56. Euclides las probó en el libro V recurriendo a la definición (V5) de la proporción por equimúltiplos.

57. Como $a+c>a$, lo que ha probado es que si $b>a$ entonces $-b<-a$. 
presentados como una mera notación (p. 308, § 481 HIPOT. 18): en la progresión geométrica $1, b, b^{2}, b^{3}, \ldots$, si se toma el término $y=b^{x}$, entonces "se llamará el exponente $x$ logaritmo del término $y[\ldots]$ escribiendo $l y=x$; suponiendo que $l$ significa logaritmo". Solo toma logaritmos respecto a una "base logarítmica» $b$ de aquellos números que son potencias de la base, $b^{x}$, con lo que las propiedades de los logaritmos resultan de manera inmediata; por ejemplo, prueba que $l^{n} \sqrt{y}=\frac{l y}{n}$ cuando $y$ es una "potencia perfecta", $y=z^{n}$, con lo que la fórmula es obvia. Lo que hace Rosell es mostrar las fórmulas generales del cálculo de logaritmos, pero viendo su validez solo en casos triviales. En el último apartado del capítulo (§ 492) explica que la teoría general de los logaritmos se basa en tablas que permitan saber el logaritmo de todos los números y muestra una tabla con los logaritmos decimales de los números 1 a 100 con siete cifras decimales, a fin de explicar someramente su estructura. Explica que, en general, los logaritmos son aproximaciones que se calculan por métodos que «no es necesario saber ahora [...] que en otro lugar tratarémos». Realiza también una breve incursión histórica por las invenciones de Neper y Briggs mencionando sus tablas y las de Ulacq, aunque indica que entre las modernas destacan las de Gardiner y las de Sherwin.

Parte II. Cap. IV. "De los principios de la Analisis algebrayca." (\$\$ 493-572, pp. 317-392)

Rosell afirma que hasta aquí ha "explicado el cálculo de las cantidades en general", pasando ahora a tratar del "Analisis algebrayca» o (p. 317, § 494 DIFIN. 48) «Arte de resolver los Problemas que se pueden proponer acerca de la cantidad por medio de equaciones». Este arte ha de atender a dos aspectos: «al método de reducir el Problema á términos algebraycos , expresandole por medio de equaciones» y a "resolver las equaciones». Tradicionalmente, el álgebra era la resolución de ecuaciones, para lo cual era necesario manipular las incógnitas y los coeficientes. Después de Newton, durante el siglo XVIII el término álgebra se fue asociando al propio "cálculo de las cantidades en general», incluyendo las extensiones de los números (negativos, irracionales, imaginarios), pero también los polinomios o los determinantes o las mismas funciones, siendo esta álgebra calculadora con cantidades cualesquiera la que se aplica a la resolución de ecuaciones, objetivo del álgebra más clásica ${ }^{58}$. Por otra parte, el término análisis, entendido en general como método para obtener el conocimiento, se concreta en la resolución de problemas matemáticos y, a partir del siglo XVI avanzado y durante el siglo XVII, el álgebra simbólica emergente llegó a ser la principal herramienta para el análisis:

58. El cambio en la imagen del álgebra está explicado en Novy, Lubos. Origins of Modern Algebra. Prage: Noordhoff, 1973, en su estudio del periodo 1770-1870. 
se realiza el análisis mediante el álgebra ${ }^{59}$. Así, Rosell incluye la resolución de ecuaciones (de primero y segundo grado) y la de sistemas lineales (de no más de tres variables) como parte del análisis para resolver problemas. El autor avisa que para resolver ecuaciones hay métodos, pero que traducir problemas comunes a ecuaciones, siempre que la cuestión planteada se preste a ello, "pende por la mayor parte, del talento é instruccion del Calculador, y solo se puede inspirar de algún modo con algunas idéas generales , y con los ejemplos que ofrecen las resoluciones de los mismos». Es, dice, como un problema de traducción que exige conocer bien las dos lenguas y los significados que expresan.

\section{Problemas de "Analisis algebrayca"}

El contenido del capítulo consiste en la resolución de los problemas 43 a 84, aunque los tres primeros no son sino consejos generales para dicha resolución; luego aparecen problemas con enunciados de situaciones de la vida real ${ }^{60}$, otros de enunciado teórico diverso y algunos que plantean directamente la resolución de ecuaciones. Los problemas están enunciados con datos concretos, pero Rosell procura resolverlos con letras que al final toman los valores prescritos. También ofrece variantes de los problemas y consideraciones sobre el método seguido que completan lo dicho en los tres primeros problemas de corte metodológico.

Hay problemas que se traducen en ecuaciones lineales, otros en sistemas lineales 2 por 2 y 3 por 3 ; otros en ecuación de segundo grado

$$
x^{2}+c x=e, \quad \text { con solución: } x=-\frac{1}{2} c \pm \sqrt{\frac{1}{4} c^{2}+e}
$$

Rosell hace una consideración sobre las raíces negativas, inconmensurables o imaginarias, afirmando que en un problema concreto estas raíces pueden indicar «diferentes grados de imposibilidad en hallar lo que se pida». Resuelve casos concretos de sistemas lineales por varios métodos de sustitución y eliminación y anuncia, sin mencionar los determinantes: «En otro lugar [...] hallarémos un método general que mas brevemente dirija á la resolucion de las equaciones del primer grado quando sean muchas las incógnitas». Hay problemas sobre progresiones

59. Véase ESPAÑOl GONZÁLEZ, Luis. "Meaning of «algebra» and «analysis» between two Encyclopedias: From the Enlightenment to the Greath War». En MARTíN-REYES, Francisco Javier; OrTEGA SALVADOR, Pedro; LOREnTE, María y González, Cristóbal (eds.). Advanced courses of mathematical analysis. Proceedings of the Sixth International School. Singapore: World Scientific, 2016, pp. 135-152. También se aplica el método analítico-algebraico a problemas de geometría, como hicieron Vieta, Descartes y también el gaditano Hugo de Omerique en su Analysis geometrica (1698), que resolvía problemas geométricos mediante las proporciones de Euclides transcritas al lenguaje algebraico simbólico. A su vez, el álgebra de los infinitesimales, esas cantidades evanescentes, fue la herramienta del análisis en la geometría trascendente.

60. Los clásicos de regla de tres, repartos, mezclas, capital e intereses, etc., también alguno más original sobre demografía. 
LUIS ESPAÑOL GONZÁLEZ Y JUNCAL MANTEROLA ZABALA

aritméticas y geométricas. Dedica un extenso problema a explicar combinatoria y los últimos problemas son de divisibilidad, resolución de ecuaciones diofánticas y de congruencias simultáneas.

\section{Balance Del aUtor y SU OBRA}

Lo mismo el autor que la obra transmiten la sensación de ser un proyecto de progreso frustrado. Recién acabada su formación universitaria, Antonio Rosell ganó con 24 años la cátedra de los nuevos Reales Estudios y se postuló como matemático profesional que buscaba una nueva orientación de la enseñanza de la matemática pura dirigida a la formación de futuros matemáticos profesionales como él, recuperando el rigor de los antiguos con el lenguaje algebraico; eran un planteamiento y un objetivo originales y avanzados en su tiempo. Antes de cumplir 40 años, empezó a padecer una enfermedad que afectó a su salud mental y le apartó de la cátedra, primero de modo temporal intermitente y de manera definitiva a los 46 años. Su dolencia ya estaba presente cuando publicó en 1785 el tomo primero de su obra principal Instituciones Matemáticas, y tuvo una incidencia manifiesta algo después. Debía completar dicha obra con un segundo tomo que ni se publicó ni nos ha llegado manuscrito. El dictamen que rechazó el Tratado de la Tactica nos aclara, sin demasiada precisión, la enfermedad que mermó al catedrático Rosell hasta la baja definitiva y ayuda también a formar un criterio acerca de la ausencia del tomo segundo de Instituciones. Los biógrafos han venido repitiendo ${ }^{61}$ que el manuscrito "quedó en manos del autor», pero si tenemos en cuenta que la pérdida de actividad en su cátedra se produjo pocos años después de publicar el primer volumen y a la vista de la naturaleza de la enfermedad que le acechó, parece lo más probable que Rosell, aun teniéndolo iniciado, no llegara nunca a completarlo; el manuscrito quedaría más bien en manos del destino que cercenó la salud y la vida matemática del autor. Para conocer someramente el contenido del tomo segundo, nos queda la descripción de sus pretensiones para la continuación que dejó en el prólogo del primero ${ }^{62}$ :

Atendiendo después á que importa enseñar quanto antes todos los principios del lenguage matemático , [...] me propongo completarle en la Geometría elementar , dando á conocer las lineas y figuras. [...] y luego paso á descubrir por todos los medios establecidos, y los que nacen de los principios de la Geometría , várias relaciones de la extension, que ademas de enseñar muchos usos prácticos, ofrecen las primeras nociones de la Geometría transcendente.

Habiendo aprendido los principiantes hasta aqui los fundamentos del lenguage matemático, y los principios de la Matemática pura, con relacion á lo restante de ella , pasarán sin tropiezo á instruirse en la ultima parte de la Analisis algebrayca ,

61. Desde Fuster y TARONCHER, op. cit.

62. Rosell Viciano (1785), op. cit., pp. XIII-XIV. 
en la Geometría transcendente, y en el Cálculo infinitesimal, que deben seguirse por este orden, con la conexion y usos que tan bien han manifestado algunos respetables Geómetras. [...] También advertirán , concluido este estudio , que las propiedades de las relaciones de las cantidades abstractas exâminadas en la Matemática pura por medio de los números, letras, signos, lineas y figuras, y aplicadas en ella á vários asuntos utiles, y freqüentes, se pueden aplicar á otros obgetos, como lo verán practicado en las Ciencias Fisico-Matemáticas.

Otra fuente de información parcial son las relaciones de los temas que forman los ejercicios públicos de sus mejores alumnos de segundo curso, pero nos dan solo enunciados teóricos o de problemas, sin información sobre el método expositivo que siguió Rosell al explicar esas cuestiones, que es donde el autor proclamaba su originalidad. El segundo tomo hubiera servido para comprender mejor el alcance de su proyecto, porque el primero es el más elemental y deja varios temas teóricos relevantes solo iniciados y con el anuncio de su posterior desarrollo en el segundo. En Instituciones la teoría de las relaciones y proporciones, que es el basamento de la obra, se presenta con una débil equiparación con la pretendida exactitud de Euclides, no obstante, hubiera sido interesante conocer el modo de abordar, desde dicho fundamento, la geometría y el cálculo infinitesimal, este último apenas vislumbrado en el interesante contenido sobre límites del primer tomo.

\section{BiBLIOGRAFÍA}

AlBiÑANA HUERTA, Salvador. La Universidad de Valencia y la ilustración en el reinado de Carlos III. Tesis doctoral. Valencia: Universidad de Valencia, 1987.

Aguilar PIÑal, Francisco. Bibliografia de autores españoles del siglo XVIII. Tomos I-X. Madrid: CSIC, Instituto Miguel de Cervantes, 1981-2001.

Caballer Vives, María Cinta. El Álgebra en la Enseñanza Secundaria en España (18361936). Tesis doctoral dirigida por Josep Llombart Palet. Leioa: UPV/EHU, 2006.

CAPEl SAez, Horacio; SÁnchez JimÉnez, Joan Eugeni y Moncada MaYa, Omar. De Palas a Minerva. La formación científica y la estructura institucional de los ingenieros militares en el siglo XVIII. Barcelona: Serval/CSIC, 1988.

Cobos Bueno, José Miguel y Vallejo Villalobos, José Ramón. «Bernardo María de Calzada y la traducción de la Lógica de Condillac en España». En GonzÁlez Redondo, Francisco de Asís (coord.). Ciencia y Técnica entre la Paz y la Guerra. 1714, 1814, 1914. Madrid: SEHCYT, 2015.

D'AlEMBERT, Jean le Rond. Discurso preliminar de la Enciclopedia. Traducido por Consuelo Berges Rábago. Madrid: Sarpe, 1984.

DE LA FuENTE, Vicente. Historia de las universidades, colegios y demás establecimientos de enseñanza en España, tomo IV. Madrid: Imprenta de la viuda é hija de Fuentenebro, 1889.

De Mendoza y Ríos, Josef María. Egercicio de Matemáticas. Madrid: Joachin Ibarra Impresor de Cámara de S. M., 1774.

Díe Fagoaga, Gonzalo. Francisco Verdejo, un mathematico olvidado. Bubok Publishing S.L., 2010. 
Dorce Polo, Carlos. Historia de las matemáticas en España. 2 vols. Prólogo de Luis Español. San Cugat: Editorial Arpegio, 2017.

ESPAÑOL GONZÁLEZ, Luis. "Meaning of "algebra» and "analysis» between two Encyclopedias: From the Enlightenment to the Greath War». En MARTín-REYES, Francisco Javier; OrTEga Salvador, Pedro; Lorente, María y González, Cristóbal (eds.). Advanced courses of mathematical analysis. Proceedings of the Sixth International School. Singapore: World Scientific, 2016.

Fuster y TARONCher, Justo Pastor. Biblioteca Valenciana de los Escritores que florecieron hasta nuestros dias y de los que aun viven. Con adiciones y enmiendas a la de D. Vicente Ximeno. Valencia: Imprenta y Librería de Ildefonso Mompié, 1830.

Gimeno Puyol, María Dolores. "Il poco numero dé libri Spagnoli": La defensa de España en las Efemeridi letterarie di Roma en el último tercio del siglo XVIII. Cuadernos de Historia Moderna, 2019, 44, 1.

GINER, Pedro Vicente. Egercicio de Matematicas. Madrid: Joachin Ibarra Impresor de Cámara de S. M., 1773.

GómEZ URDÁÑEZ, José Luis. El proyecto reformista de Ensenada. Lleida: Ed. Milenio, 1996.

JACOBS, Helmut C. «Utopías de la organización e institucionalización de las artes y ciencias en la época de la ilustración: Los proyectos de fundación de academias». Anejos de Dieciocho, 2019, 5, Spring. Universität Duisburg-Essen.

Manterola Zabala, María Juncal. Las matemáticas en los estudios de náutica en España en el siglo XVIII: estudio comparativo de los libros de texto empleados en la formación de pilotos y guardiamarinas. Tesis doctoral dirigida por Luis Español e Itsaso Ibáñez. Logroño: Universidad de La Rioja, 2016.

Memorial Literario, Instructivo y Curioso de la Corte de Madrid, tomo XI, número LXI. Madrid: Imprenta Real, 1787.

Mercurio de España, agosto de 1786. Madrid: Imprenta Real.

Novy, Lubos. Origins of Modern Algebra. Prage: Noordhoff, 1973.

Novy, Lubos. «Las matemáticas en la enciclopedia de Diderot y d'Alembert». Llull, Revista de la SEHCYT, 1993, 16, 30.

Rosell Viciano, Antonio Gregorio. Demonstracion de las causas que concurrieron á los daños y ruinas de las obras del Prado nuevo de Madrid en la tarde del 23 de Setiembre de 1775, y modo de precaverlas en adelante. Madrid: Imprenta de Ibarra, 1775.

Rosell Viciano, Antonio Gregorio. La Geometría de los Niños. Madrid: Imprenta Real, 1784.

Rosell Viciano, Antonio Gregorio. Instituciones Matemáticas. Madrid: Imprenta Real, 1785.

SEMPERE y GUARINOS, Juan. Ensayo de una biblioteca española de los mejores escritores del reinado de Carlos III, tomo V. Madrid: Imprenta Real, 1789.

Simón Díaz, José. Historia del Colegio Imperial de Madrid, tomo II. Madrid: Instituto de Estudios Madrileños, CSIC, 1959.

Simón Díaz, José. "Los Reales Estudios de San Isidro: Nuevas noticias". Anales del Instituto de Estudios Madrileños. Madrid: Instituto de Estudios Madrileños, CSIC, 1973, IX.

VIÑAO Frago, Antonio. "Disciplinas académicas y profesionalización docente: los Reales Estudios de San Isidro (1770-1808)». En Fell, Ève-Marie y GuereÑa, Jean-Louis (dirs.). L'Université en Espagne et en Amérique Latine du Moyen Âge à nos jours. II: Enjeux, contenus, images. Tours: Presses universitaires François-Rabelais, 1998. Edición disponible en línea (generada el 16 de abril de 2021). http://books.openedition.org/ pufr/5982 
